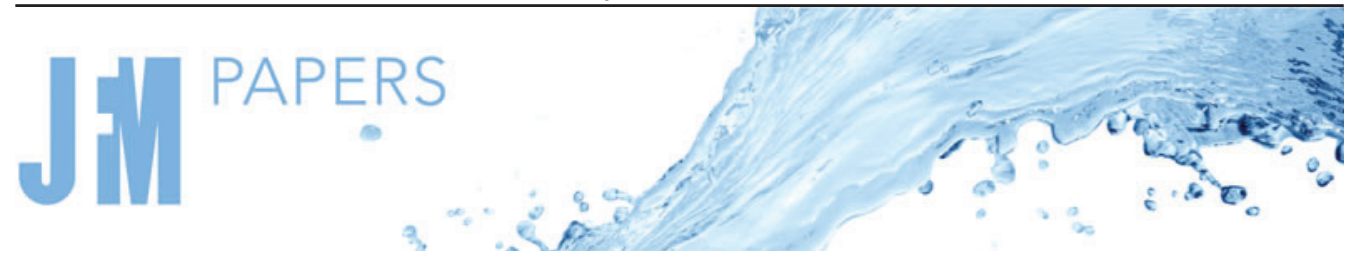

\title{
A linear stability analysis of two-layer moist convection with a saturation interface
}

\author{
Hao Fu† \\ Department of Earth System Science, Stanford University, Stanford, CA 94305, USA
}

(Received 2 September 2020; revised 28 June 2021; accepted 2 September 2021)

The linear convective instability of a mixture of dry air, water vapour and liquid water, with a stable unsaturated layer residing on an unstable saturated layer, is studied. It may serve as a prototype model for understanding the instability that causes mixing at the top of stratocumulus cloud or fog. Such a cloud-clear air interface is modelled as an infinitely thin saturation interface where radiative and evaporative cooling take place. The interface position is determined by the Clausius-Clapeyron equation, and can undulate with the evolution of moisture and temperature. In the small-amplitude regime two physical mechanisms are revealed. First, the interface undulation leads to the undulation of the cooling source, which destabilizes the system by superposing a vertical dipole heating anomaly on the convective cell. Second, the evolution of the moisture field induces non-uniform evaporation at the interface, which stabilizes the system by introducing a stronger evaporative cooling in the ascending region and vice versa in the descending region. These two mechanisms are competing, and their relative contribution to the instability is quantified by theoretically estimating their relative contribution to buoyancy flux tendency. When there is only evaporative cooling, the two mechanisms break even, and the marginal stability curve remains the same as the classic two-layer Rayleigh-Bénard convection with a fixed cooling source.

Key words: moist convection, atmospheric flows, buoyancy-driven instability

\section{Introduction}

A thermally unstable layer lying over a solid surface and below a stable layer renders a two-layer convection set-up that is frequently seen in geophysical flow. For a mixture of dry air and water such as the atmosphere, a special case is when the lower layer is saturated to water vapour (cloudy), with liquid water suspending in the air as droplets.

$†$ Email address for correspondence: haofu@stanford.edu

(C) The Author(s), 2021. Published by Cambridge University Press. This is an Open Access article, distributed under the terms of the Creative Commons Attribution licence (https://creativecommons. org/licenses/by/4.0/), which permits unrestricted re-use, distribution, and reproduction in any medium, provided the original work is properly cited. 


\section{H. Fu}

The macroscopic cloud-clear air interface is a thin saturation interface. It is susceptible to evaporative cooling due to the molecular diffusion and turbulent mixing between the unsaturated and saturated parcels, as well as longwave radiative cooling due to the divergence of radiative fluxes caused by the abrupt change of the longwave absorption coefficient (Mellado 2017). The cooling at the interface drives turbulence, which entrains the upper-layer dry air into the cloud layer. This is an important factor that leads to the breakup of stratocumulus and fog (Lilly 1968). The fog is simply the cloud that touches the sea or land surface (Emanuel 1994). The breakup is not only important for aviation which requires an accurate prediction of visibility, but also for understanding the cloud-radiation feedback that impacts the climate (Cotton, Bryan \& van den Heever 2011; Schneider, Kaul \& Pressel 2019). Despite its importance, the cloud-top entrainment is still poorly understood. A primary difficulty is that the mixing between the cloud and dry air is nonlinear - a denser mixture, or a buoyancy reversal, could be produced (Lilly 1968; Randall 1980). When the buoyancy reversal is strong enough, it can drive more turbulence and cause further entrainment, leading to runaway instability (Shy \& Breidenthal 1990). Such a buoyancy reversal can be enhanced by cloud-top radiative cooling (Lilly 1968; Sayler \& Breidenthal 1998; de Lozar \& Mellado 2013). Other factors such as vertical shear (Schulz \& Mellado 2018) and droplet settling (Schulz \& Mellado 2019) further complicate the problem.

The cloud-top mixing has been studied with the idealized parcel model (Randall 1980), direct numerical simulation (Siems et al. 1990; Siems \& Bretherton 1992; Mellado et al. 2009; Mellado González 2010; de Lozar \& Mellado 2015; Schulz \& Mellado 2018, 2019) and the laboratory water tank which uses the nonlinear dependence of density on chemical concentrations to mimic evaporative cooling (Turner \& Yang 1963; Shy \& Breidenthal 1990). Randall (1980) proposed a necessary condition for self-sustaining entrainment with parcel argument: the above-cloud parcel, after being cooled to saturation by isobaric mixing with the in-cloud air, must be less buoyant than the in-cloud air. Shy \& Breidenthal (1990) used the tank experiment to show that an even denser mixture is needed, because the eddy produced by the mixture needs to do work against the stable stratification to sustain the entrainment. Siems et al. (1990) further studied the role of large eddies with numerical simulation. They considered the evaporative production of a thin very dense layer at the interface of a uniformly dense lower layer and a uniformly light upper layer. Two processes are found to enhance entrainment. First, in the laminar regime, the strong diffusive evaporation at the updraft plume's front ('flame front') generates cold air. There, the deformation field enhances diffusion by squeezing the contour surfaces of the 'dry air mixing fraction' (a linear function of enthalpy or total water content). The dense interfacial air is gravitationally unstable, so it tilts the interface, and then slips from the interfacial ridge where the 'flame front' resides to the interfacial valley, producing a concentrated downdraft. Second, in the turbulent regime, the vortex ring associated with the updraft plume can engulf the dry air into the cloud and induce mixing. Mellado et al. (2009) used an analytical three-layer Rayleigh-Taylor instability model to quantify the interface undulation in the first process, with the densest middle layer existing at the beginning.

However, how does the dense layer form? And how does it couple with the in-cloud overturning eddy? The in-cloud overturning eddy is cooperatively driven by the cloud-top and in-cloud instability, so the extra evaporation at the 'flame front' must have an influence on it. Because the diffusional evaporation is non-uniform at the interface due to the overturning eddy, a non-diffusive Rayleigh-Taylor type model is not enough to answer this question. A Rayleigh-Bénard convection model with its upper boundary serving as the undulating interface, and with the heat flux there depending on fluid motion, was suggested by Mellado et al. (2009). 


\section{Saturation interface instability}

Enlightened by this, we build a two-layer Rayleigh-Bénard convective instability model that, for the first time, couples the moist thermodynamics, as well as the interface and the in-cloud cell. To facilitate the linear stability analysis, the idealized set-up of Siems et al. (1990) is modified by changing the basic state from a local staircase buoyancy reversal at the interface to a global piecewise linear buoyancy distribution across the domain. In other words, the cloud-top buoyancy reversal layer is extended to the whole domain, and the in-cloud unstable stratification is simply part of it. The lower rigid lid, which does not exist for the real cloud-top mixing layer, artificially set a length scale for the largest overturning eddy (Siems et al. 1990). It may also qualitatively represent the cloud bottom, or the surface of a fog layer, but not in a strict sense due to their multiscale and turbulent nature. We refer the reader to a series of works on the 'mesoscale entrainment instability' that use a parameterized entrainment rate to model this larger-scale scenario (Fielder 1984; Breidenthal \& Baker 1985; Yano 2021). In addition, we use a Dirac-delta function to represent radiative cooling at the infinitely thin interface and study how it cooperates with evaporative cooling in this linear stability problem.

Technically, this instability model is an update of the classic two-layer Rayleigh-Bénard convection, which is the simplest model for studying penetrative convection (Gribov \& Gurevich 1957). The basic state is the balance between diffusion and a fixed horizontally uniform thin cooling source (or heating source such as a light beam if the system is upside down), which yields a piecewise linear vertical profile of buoyancy (Whitehead \& Chen 1970). Ogura \& Kondo (1970) used linear stability analysis to show that a stronger upper-layer stratification suppresses convection. It will be shown that the inclusion of phase change and radiative cooling only changes the matching condition in the stability analysis. The result is a more confined lower-layer convective cell and a stronger upper-layer secondary cell.

The moist convection with a saturation interface can be compared with another more intensely investigated phenomenon: convection of two-phase-one-component flow, such as a liquid-gas system (Busse \& Schubert 1971; Hsieh 1972; McFadden et al. 2007; Konovalov, Lyubimov \& Lyubimova 2017). Latent heat is absorbed or released at the fluctuating interface which is also not a material surface. There are two main differences.

(i) The interface of a two-phase-one-component flow is completely determined by pressure and temperature via the Clausius-Clapeyron equation. The moist air problem, on the other hand, is a two-phase-two-component flow, whose saturation interface depends mainly on temperature and water content, and hardly on the pressure anomaly.

(ii) The phase transition rate at the liquid-gas interface is proportional to mass flux which is zero at the diffusive-equilibrium basic state. The moist air problem is more complicated because it involves liquid droplets. In a very idealized case which will be discussed, its evaporation rate at the interface only depends on the liquid water diffusive flux which is non-zero at the basic state (Bretherton 1987).

Though the physics is quite different, some of the methodology in analysing the two-phase problem is employed in this model, as will be mentioned in the context.

The paper is organized in the following way. Section 2 introduces the physical model. Section 3 introduces the linear stability analysis. Section 4 introduces the neutral stability curve, eigenfunction of the neutral mode and discusses the instability mechanism. Section 5 uses nonlinear numerical simulation to benchmark the linear stability analysis and briefly explores the finite-amplitude regime. Section 6 concludes the paper. 


\section{The physical model}

\subsection{The governing equations}

A two-layer set-up with idealized radiation and moist processes is built. It is modified from the fully nonlinear model of Siems et al. (1990) and Mellado et al. (2009) by adding a Dirac-delta function radiative cooling, as well as changing the basic state buoyancy profile from a local staircase reversal to a global piecewise linear one. The governing equations are expressed with explicit temperature and water species, rather than the mixing fraction formulation. The following four processes are omitted.

(i) The background vertical shear which enhances the interfacial mixing (Schulz \& Mellado 2018).

(ii) The droplet settling which reduces the interfacial evaporation by removing droplets near the cloud top (Bretherton, Blossey \& Uchida 2007; Schulz \& Mellado 2019).

(iii) The lightening effect of water vapour and the loading of liquid water on buoyancy (Austin 1995).

(iv) The adiabatic expansion and compression of a parcel in vertical motion which is only significant for a sufficiently deep domain, such as the whole stratocumulus layer (Siems et al. 1990).

The symbol of all the dimensional variables (but not dimensional parameters) will include a '*'. The basic state saturation interface is at $z^{*}=0$, the lower boundary is a rigid lid at $z^{*}=-H$ and the upper layer is infinitely deep. Figure 1 shows a schematic diagram of the set-up. The flow is assumed to be Boussinesq. The continuity equation, momentum equation, Clausius-Clapeyron equation, water vapour diagnostic equation, total water equation and thermodynamic equation are

$$
\begin{gathered}
\nabla^{*} \cdot \boldsymbol{u}^{*}=0 \\
\frac{\partial \boldsymbol{u}^{*}}{\partial t^{*}}+\boldsymbol{u}^{*} \cdot \nabla^{*} \boldsymbol{u}^{*}=-\frac{1}{\rho_{0}} \nabla^{*} p^{*}+\frac{g}{T_{0}} T^{*} \boldsymbol{k}+v \nabla^{* 2} \boldsymbol{u}^{*}, \\
q_{v s}^{*}\left(T^{*}\right)=q_{v s 0}+\tilde{\lambda}\left(T^{*}-T_{0}\right), \\
q_{v}^{*}=\min \left\{q_{v s}^{*}, q_{t}^{*}\right\}, \\
\frac{\partial q_{t}^{*}}{\partial t^{*}}+\boldsymbol{u}^{*} \cdot \nabla^{*} q_{t}^{*}=\kappa \nabla^{* 2} q_{t}^{*} \quad \text { with } q_{t}^{*}=q_{v}^{*}+q_{l}^{*}, \\
\frac{\partial T^{*}}{\partial t^{*}}+\boldsymbol{u}^{*} \cdot \nabla^{*} T^{*}-\kappa \nabla^{* 2} T^{*}= \\
{\left[Q_{\text {rad }}^{*}+\left.\frac{L_{v} \kappa}{c_{p}} \nabla^{*} q_{l}^{*}\right|_{z^{*}=z_{s}^{*-}} \cdot \boldsymbol{n} \delta\left(z^{*}-z_{s}^{*}\right)\right]} \\
\times \delta\left(z^{*}-z_{s}^{*}\right),
\end{gathered}
$$

where $\boldsymbol{u}^{*}=u^{*} \boldsymbol{i}+v^{*} \boldsymbol{j}+w^{*} \boldsymbol{k}$ is the three-dimensional velocity vector, $\boldsymbol{i}, \boldsymbol{j}$ and $\boldsymbol{k}$ are the three unit vectors of Cartesian coordinate, $\boldsymbol{x}^{*}=x^{*} \boldsymbol{i}+y^{*} \boldsymbol{j}+z^{*} \boldsymbol{k}$ is the position vector, $\nabla^{*}=\boldsymbol{i} \partial / \partial x^{*}+\boldsymbol{j} \partial / \partial y^{*}+\boldsymbol{k} \partial / \partial z^{*}$ is the gradient operator, $t^{*}$ is time and $\min \{\}$ is the minimum operator.

The variable $p^{*}$ is perturbation pressure, $T^{*}$ is temperature, $q_{v s}^{*}$ is the saturation vapour content (the mass of saturated vapour in $1 \mathrm{~kg}$ of air), $q_{t}^{*}$ is the total water content (the total water mass in $1 \mathrm{~kg}$ of air) which is the sum of vapour content $q_{v}^{*}$ and liquid water content $q_{l}^{*}$. The constants $\rho_{0}$ is the reference density, $v$ is the kinematic viscosity, $\kappa$ is the diffusivity shared by $T^{*}$ and all water species, $L_{v}$ is the evaporation latent heat and $c_{p}$ is the isobaric specific heat. For applications to stratocumulus, $v$ and $\kappa$ should be viewed 
(a)



(b)



Figure 1. A schematic diagram of the two-layer set-up with $(a)$ the dimensional values and $(b)$ the non-dimensional values. The solid red lines denote temperature profiles, and the solid blue lines denote total water content profiles. The dashed black line denotes the basic state saturation interface. The light blue shadow denotes the saturated region. The deep blue shadow denotes the vertical profile of the liquid water content $q_{l}$ whose basic state non-dimensional value is $1-\lambda$ at $z=-1$ and 0 at $z=0$.

as eddy viscosity and diffusivity, respectively. The saturated water vapour content $q_{v s}^{*}$ is linearized to $T^{*}$ (Randall 1980; Spyksma, Bartello \& Yau 2006), with $\tilde{\lambda}$ as the slope, $T_{0}$ and $q_{v s 0}$ as the reference temperature and saturated water vapour content. They are the radiative-diffusive equilibrium value at $z^{*}=0$. The constant $Q_{\text {rad }}^{*}$ is the longwave radiative cooling strength (strictly speaking, the radiative flux density expressed in temperature) that depends on the Stefan-Boltzmann law. It is the small penetration depth limit of the simple radiation transfer model of de Lozar \& Mellado (2013). The validity of this radiative representation is discussed in Appendix A. The vector $\boldsymbol{n}$ is the unit vector perpendicular to the saturation interface and points from the saturated region to the unsaturated region. The superscript ' - ' in $z_{s}^{*-}$ means a tiny distance below $z_{s}^{*}$, and vice versa for ' + ' which will appear later.

The adjustment to thermodynamic equilibrium is assumed to be instantaneous, so the supersaturation (Chandrakar et al. 2020) is not allowed, as shown in (2.4). The assumption of identical diffusivity for $T^{*}, q_{v}^{*}, q_{l}^{*}$ and $q_{t}^{*}$ was proposed by Bretherton (1987), and has been used in many cloud-top mixing simulations (Siems et al. 1990; Mellado et al. 2009; Mellado González 2010; de Lozar \& Mellado 2015; Schulz \& Mellado 2018). It leads to a drastic simplification: evaporative cooling is determined by the liquid water diffusive flux at the interface. An equivalent argument is shown in (8) of de Lozar \& Mellado (2015) in their mixing fraction formulation. If the diffusivity of temperature and water species are different, diffusion will cause a phase change inside the saturated region. In the real atmosphere the dispersion of droplets is much more complicated than Fickian diffusion (Bois \& Kubicki 2003).

\subsection{Boundary condition}

The boundary condition is identical to the simulation of Siems et al. (1990) and Mellado et al. (2009), except for an infinitely deep upper layer (rather than an upper lid). The lower boundary is a free-slip rigid lid with fixed temperature $T^{*}$ and total water content $q_{t}^{*}$,

$$
\left.\begin{array}{c}
\left.\frac{\partial u^{*}}{\partial z^{*}}\right|_{z^{*}=-H}=\left.\frac{\partial v^{*}}{\partial z^{*}}\right|_{z^{*}=-H}=\left.w^{*}\right|_{z^{*}=-H}=0, \\
\left.T^{*}\right|_{z^{*}=-H}=T_{0}+\Delta T,\left.\quad q_{t}^{*}\right|_{z^{*}=-H}=q_{v s}+\Delta q_{t},
\end{array}\right\}
$$




\section{H. Fu}

where $\Delta T$ and $\Delta q_{t}$ are the temperature and water content drop across the saturated layer, respectively, and are both positive. In the real atmosphere the downward penetration depth of the mixture produced at the cloud top is limited by its moisture content. The adiabatic compression can make it dry out and then quickly gain buoyancy in the dry adiabatic process, even before reaching the cloud bottom (Siems et al. 1990). This effect, which is not explicitly considered in this model, can be implicitly carried by the lower lid. At $z^{*} \rightarrow \infty$, the velocity vanishes, and $T^{*}$ and $q_{t}^{*}$ asymptotically approach a reference linear profile,

$$
\left.\begin{array}{l}
\left.u^{*}\right|_{z^{*} \rightarrow \infty}=\left.v^{*}\right|_{z^{*} \rightarrow \infty}=\left.w^{*}\right|_{z^{*} \rightarrow \infty}=0, \\
\left.T^{*}\right|_{z^{*} \rightarrow \infty}=T_{0}-\Gamma_{T \infty} z,\left.\quad q_{t}^{*}\right|_{z^{*} \rightarrow \infty}=q_{v s 0}-\Gamma_{q \infty} z,
\end{array}\right\}
$$

where $\Gamma_{T \infty}$ (negative) and $\Gamma_{q \infty}=\Delta q_{t} / H$ (positive) are the lapse rate of $T^{*}$ and $q_{t}^{*}$ at $z^{*} \rightarrow \infty$. Strictly speaking, the upper-layer thickness should be smaller than $q_{v s 0} / \Gamma_{q \infty}$ to make $q_{t}^{*}$ positive. In the real atmosphere $\Gamma_{q \infty}$ is changing with height, and is small outside of the cloud-top mixing layer (Mellado 2017). In this model, switching the water lapse rate to a small value above the convective penetration depth $H_{p}$ should not influence the result. Thus, we only require $q_{t}^{*}$ to be still positive at $z^{*}=H_{p}$. Considering that $H_{p}$ is the neutrally buoyant level of a parcel which ascends adiabatically from the lower boundary, we get $H_{p} \sim-\Delta T / \Gamma_{T \infty}$. This poses a physical constraint on our model: $H_{p} \ll q_{v s 0} / \Gamma_{q \infty}$ or $-\Delta T / \Gamma_{T \infty} \ll q_{v s 0} / \Gamma_{q \infty}$. Furthermore, as $q_{v s 0}$ only influences the basic state and does not enter the linear stability analysis, we can always choose a $q_{v s 0}$ to satisfy this physical constraint.

\subsection{The non-dimensional group}

The basic state is in radiative-diffusive equilibrium, without any motion. The basic state variables are denoted with an overline. The Dirac-delta function cooling at the saturation interface makes the basic state temperature profile $\overline{T^{*}}$ piecewise linear, which decreases with height in the lower saturated layer and increases with height in the upper unsaturated layer. The basic state water content $\overline{q_{t}^{*}}$ decreases linearly with height. These basic state profiles will be used to non-dimensionalize the equations.

The governing equation of $\overline{q_{t}^{*}}$ is obtained from (2.5). Further using the boundary condition in (2.7) and (2.8), we get

$$
-\kappa \frac{\mathrm{d}^{2} \overline{q_{t}^{*}}}{\mathrm{~d} z^{* 2}}=0 \Rightarrow \overline{q_{t}^{*}}(z)=q_{v s 0}-\Gamma_{q \infty} z^{*} .
$$

The governing equation of $\overline{T^{*}}$ and its solution are obtained with (2.3), (2.4), (2.6) and (2.9),

$$
\begin{aligned}
-\kappa \frac{\mathrm{d}^{2} \overline{T^{*}}}{\mathrm{~d} z^{* 2}} & =\left(Q_{\text {rad }}^{*}+\left.\frac{L_{v} \kappa}{c_{p}} \frac{\mathrm{d} \overline{q_{l}^{*}}}{\mathrm{~d} z^{*}}\right|_{z^{*}=0^{-}}\right) \delta\left(z^{*}\right), \\
\overline{T^{*}}\left(z^{*}\right) & =\left\{\begin{array}{ll}
T_{0}-\Gamma_{T \infty} z^{*}, & 0 \leq z^{*}<\infty, \\
T_{0}-\Gamma_{T s} z^{*}, & -H<z^{*} \leq 0,
\end{array}\right\}
\end{aligned}
$$




\section{Saturation interface instability}

where $\Gamma_{T s}=\Delta T / H$ (positive) is the $\overline{T^{*}}$ lapse rate in the saturated layer. The parameters $\Gamma_{T s}$ and $\Gamma_{T \infty}$ are linked with the radiative and evaporative cooling rate at the interface:

$$
\Gamma_{T_{s}}=\Gamma_{T \infty}-\frac{1}{\kappa}\left[Q_{\text {rad }}^{*}-\frac{L_{v} \kappa}{c_{p}} \Gamma_{q \infty}\left(1-\frac{\tilde{\lambda} \Delta T}{\Delta q_{t}}\right)\right] .
$$

We choose $\Delta T$ and $\Delta q_{t}$ as the temperature and water content scale, the saturated layer depth $H$ as the length scale and $H^{2} / v$ as the time scale. The dimensionless variables (without '*') obey

$$
\left.\begin{array}{c}
\boldsymbol{x}^{*}=\boldsymbol{x} H, z_{s}^{*}=z_{s} H, t^{*}=t H^{2} / v, \\
\boldsymbol{u}^{*}=\boldsymbol{u} v / H, p^{*}=p \rho_{0} v^{2} / H^{2}, T^{*}-T_{0}=T \Delta T, \\
q_{t}^{*}-q_{v s 0}=q_{t} \Delta q_{t}, q_{l}^{*}-q_{v s 0}=q_{l} \Delta q_{t}, q_{v}^{*}-q_{v s 0}=q_{v} \Delta q_{t}, q_{v s}^{*}-q_{v s 0}=q_{v s} \Delta q_{t} .
\end{array}\right\}
$$

The problem is controlled by five non-dimensional parameters:

$$
\left.\begin{array}{r}
R a=\frac{g\left(\Delta T / T_{0}\right) H^{3}}{\nu \kappa}, \operatorname{Pr}=\frac{v}{\kappa}, \\
M=\frac{L_{v} \Delta q_{t}}{c_{p} \Delta T}, Q_{r a d}=\frac{Q_{r a d}^{*}}{\Delta T \nu / H} \quad \text { and } \quad \lambda=\frac{\tilde{\lambda} \Delta T}{\Delta q_{t}} .
\end{array}\right\}
$$

Rayleigh number $R a$ measures the relative strength of the destabilizing effect of unstable stratification and the stabilizing effect of viscosity and temperature diffusivity. Prandtl number $\operatorname{Pr}$ is the ratio of viscosity to temperature and water diffusivity. The $Q_{\text {rad }}$ measures the contribution of radiative cooling.

Now, we consider $\lambda$ and $M$ that involve the moisture effect. It is worth noting that their ranges are constrained. The parameter $\lambda$ represents the Clausius-Clapeyron equation which influences the interface height $z_{s}$, as well as the partition between $q_{v}$ and $q_{l}$ in the saturated layer which is purely diagnostic under the thermodynamic equilibrium assumption. There must be $\lambda<1$ to guarantee the lower layer is saturated, and $\lambda>0$ to guarantee the monotonicity of saturated vapour pressure on $T$. The parameter $M$ is the ratio of latent to dry enthalpy change across the saturated layer. It can be represented as $M=\left(L_{v} / c_{p}\right) \tilde{\lambda} \lambda^{-1}$. The parameter $\left(L_{v} / c_{p}\right) \tilde{\lambda}$ ranges from 1 to 2 in the $280-290 \mathrm{~K}$ temperature range at $900 \mathrm{hPa}$ level which represents the stratocumulus (Randall 1980). This, together with $0<\lambda<1$, indicates $M \gtrsim 1$.

Before linearizing the problem, the basic state non-dimensional profiles $\bar{T}, \overline{q_{t}}, \overline{q_{v s}}$ and $\overline{q_{l}}$ are derived from their dimensional expression. The cooling strength at the interface can be further depicted with a parameter $\gamma_{T}$ which measures the temperature gradient ratio of the upper and lower layers:

$$
\gamma_{T} \equiv \frac{\left(\mathrm{d} \overline{T^{*}} / \mathrm{d} z^{*}\right)_{u}}{\left(\mathrm{~d} \overline{T^{*}} / \mathrm{d} z^{*}\right)_{s}}=\frac{\Gamma_{T \infty}}{\Gamma_{T s}} .
$$

Here ()$_{u}$ and ()$_{s}$ denote the unsaturated (upper) and saturated (lower) layer property, respectively. The parameter $\gamma_{T}$ is negative in our case where temperature decreases with height in the lower layer and increases with height in the upper layer. Using (2.3), (2.9), 
(2.10), (2.12) and (2.14), we get

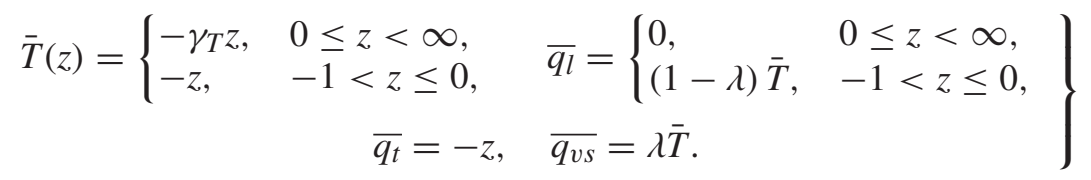

The parameter $\gamma_{T}$ can be linked to the radiative cooling rate $Q_{\text {rad }}$ and the basic state evaporative cooling rate $\overline{Q_{\text {evap }}}$ by substituting (2.11) into (2.14), and using the non-dimensional treatment in (2.12) and (2.13):

$$
\overline{Q_{\text {evap }}}+Q_{\text {rad }}=-\frac{1-\gamma_{T}}{P r} \quad \text { with } \overline{Q_{\text {evap }}}=-\frac{M(1-\lambda)}{P r} .
$$

\subsection{The linearized governing equation}

The disturbance non-dimensional temperature and water content is obtained by subtracting their basic state values from their total values, i.e.

$$
T^{\prime}=T-\bar{T}, \quad q_{t}^{\prime}=q_{t}-\overline{q_{t}}, \quad q_{l}^{\prime}=q_{l}-\overline{q_{l}}, \quad q_{v}^{\prime}=q_{v}-\overline{q_{v}}, \quad q_{v s}^{\prime}=q_{v s}-\overline{q_{v s}} .
$$

The non-dimensional gradient operator is defined as $\nabla=\boldsymbol{i} \partial / \partial x+\boldsymbol{j} \partial / \partial y+\boldsymbol{k} \partial / \partial z$. With (2.12), (2.13), (2.15) and (2.17a-e), the dimensional equations (2.1)-(2.6) are non-dimensionalized and linearized,

$$
\begin{gathered}
\nabla \cdot \boldsymbol{u}=0 \\
\frac{\partial \boldsymbol{u}}{\partial t}=-\nabla p+\frac{R a}{\operatorname{Pr}} T^{\prime} \boldsymbol{k}+\nabla^{2} \boldsymbol{u} \\
\left.q_{t}^{\prime}\right|_{z=0}+\left.z_{s} \frac{\mathrm{d} \overline{q_{t}}}{\mathrm{~d} z}\right|_{z=0}=\lambda\left(\left.T^{\prime}\right|_{z=0^{-}}+\left.z_{s} \frac{\mathrm{d} \bar{T}}{\mathrm{~d} z}\right|_{z=0^{-}}\right)=\lambda\left(\left.T^{\prime}\right|_{z=0^{+}}+\left.z_{s} \frac{\mathrm{d} \bar{T}}{\mathrm{~d} z}\right|_{z=0^{+}}\right), \\
\frac{\partial T_{l}^{\prime}=[1-\mathcal{H}(z)]\left(q_{t}^{\prime}-\lambda T^{\prime}\right),}{\frac{1-\gamma_{T}}{\partial t} z_{s} \frac{\mathrm{d} \delta(z)}{\mathrm{d} z}+\frac{M}{\operatorname{Pr}}\left(\left.\frac{\partial q_{t}^{\prime}}{\partial z}\right|_{z=0}-\left.\lambda \frac{\partial T^{\prime}}{\partial z}\right|_{z=0^{-}}\right) \delta(z)+\frac{1}{\operatorname{Pr}} \nabla^{2} T^{\prime}} \\
+w\left[1-\left(1-\gamma_{T}^{\prime}\right) \mathcal{H}(z)\right],
\end{gathered}
$$

where $\mathcal{H}(z)$ is the Heaviside function. The variables $q_{t}^{\prime}, q_{v s}^{\prime}, q_{l}^{\prime}, T^{\prime}, \boldsymbol{u}$ and $z_{s}$ all have small amplitude. The non-dimensional boundary conditions are obtained from (2.7) and (2.8),

$$
\left.\begin{array}{rl}
\frac{\partial u}{\partial z} & =\frac{\partial v}{\partial z}=w=q_{t}^{\prime}=q_{l}^{\prime}=T^{\prime}=0, \quad z=-1 \\
u & =v=w=q_{t}^{\prime}=q_{l}^{\prime}=T^{\prime}=0, \quad z \rightarrow \infty
\end{array}\right\}
$$

Three remarks on the governing equations are made below.

First, the $\mathrm{d} \delta(z) / \mathrm{d} z$ term on the right-hand side of (2.23) indicates that there is a discontinuity of the small-amplitude $T^{\prime}$ at $z=0$, which is strictly proved later in (3.6). 

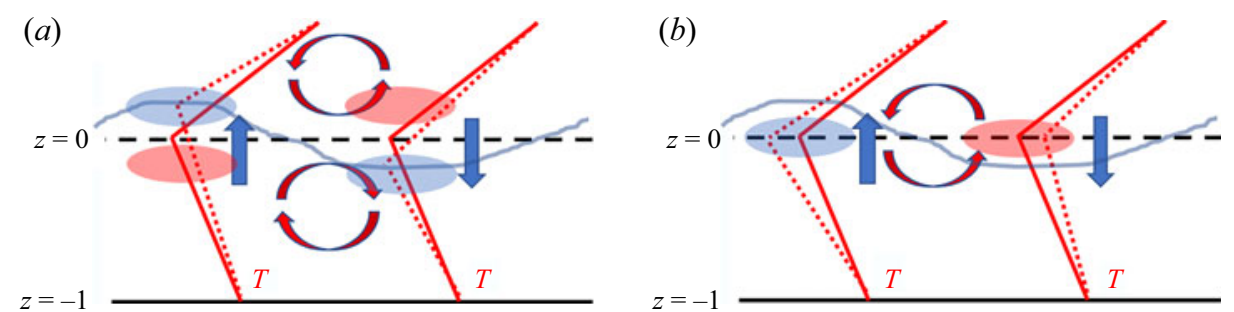

Figure 2. A schematic diagram of $(a)$ the effect of the undulating interface and $(b)$ the effect of non-uniform evaporation at the interface. The solid blue line denotes the perturbed saturation interface. The dashed black line denotes the basic state saturation interface. The vertical blue arrows denote the vertical motion at the interface. The blue patch denotes the cooling anomaly and the red patch denotes the warming anomaly. The circulating red arrows in $(a, b)$ denote the baroclinic torque produced by the interface undulation and non-uniform evaporation, respectively. The solid red lines denote the basic state temperature profiles $(\bar{T})$, and the dotted red lines denote the temperature profiles changed by the interface undulation or the non-uniform evaporation alone.

Physically, it is because the shift of the cooling source produces a small amplitude yet systematic deviation of the temperature profile from the basic state, as illustrated in figure $2(a)$. The finite-amplitude $T^{\prime}$ is continuous everywhere, with a sharp transition within $z \in\left(-z_{s}, z_{s}\right)$. The thickness of the $T^{\prime}$ transition region reduces to the thickness of the saturation interface at the small-amplitude limit, which is infinitely thin.

Second, (2.21) is derived by first transforming (2.4) to $q_{l}=\left[1-\mathcal{H}\left(z-z_{s}\right)\right]\left(q_{t}-q_{v s}\right)$, and then decomposing it into the basic state and perturbation variables

$$
\begin{aligned}
\overline{q_{l}}+q_{l}^{\prime} & =\left[1-\mathcal{H}\left(z-z_{s}\right)\right]\left(\overline{q_{t}}+q_{t}^{\prime}-\overline{q_{v s}}-q_{v s}^{\prime}\right) \\
& \approx\left[1-\mathcal{H}(z)+z_{s} \delta(z)\right]\left(\overline{q_{t}}+q_{t}^{\prime}-\lambda \bar{T}-\lambda T^{\prime}\right) \\
& \approx[1-\mathcal{H}(z)]\left(\overline{q_{t}}-\lambda \bar{T}\right)+[1-\mathcal{H}(z)]\left(q_{t}^{\prime}-\lambda T^{\prime}\right)+z_{s} \delta(z)\left(\overline{q_{t}}-\lambda \bar{T}\right),
\end{aligned}
$$

where we have used $\overline{q_{v s}}=\lambda \bar{T}$ in (2.15) and $q_{v s}^{\prime}=\lambda T^{\prime}$, as well as the Taylor expansion of the Heaviside function $\mathcal{H}\left(z-z_{s}\right) \approx \mathcal{H}(z)-z_{s} \delta(z)$. Because $\overline{q_{t}}-\lambda \bar{T}=0$ at $z=0$, the third term on the right-hand side of $(2.25)$ vanishes. Because $\overline{q_{l}}=[1-\mathcal{H}(z)]\left(\overline{q_{t}}-\lambda \bar{T}\right)$ as is indicated by (2.15), the perturbation part of (2.25) becomes (2.21).

Third, (2.23) is derived by first linearizing $\left.\nabla q_{l}\right|_{z=z_{s}^{-}} \cdot \boldsymbol{n}$ to $\partial q_{l} /\left.\partial z\right|_{z=z_{s}^{-}}$, and then using Taylor expansion of the Dirac-delta function $\delta\left(z-z_{s}\right) \approx \delta(z)-z_{s} \mathrm{~d} \delta(z) / \mathrm{d} z$ to linearize the interfacial cooling term around $z=0$. The derivative and Taylor expansion of a Dirac-delta function is defined by considering it as a generalized function (a distribution). See appendix C.4 of the book of Pope (2000) for a reference. The Taylor expansion of the Heaviside function also exists, because it is the integral of the Taylor expansion of a Dirac-delta function. The detail of the expansion of the Dirac-delta function term in the non-dimensional version of (2.6) is as follows:

$$
\begin{aligned}
& \left(Q_{\text {rad }}+\left.\frac{M}{P r} \nabla q_{l}\right|_{z=z_{s}^{-}} \cdot n\right) \delta\left(z-z_{s}\right) \\
& \approx\left[Q_{r a d}+\frac{M(1-\lambda)}{P r} \frac{\mathrm{d} \overline{q_{t}}}{\mathrm{~d} z}+\left.\frac{M}{\operatorname{Pr}} \frac{\partial q_{l}^{\prime}}{\partial z}\right|_{z=0^{-}}\right]\left(\delta(z)-z_{s} \frac{\mathrm{d} \delta(z)}{\mathrm{d} z}\right) \\
& \approx\left[Q_{\text {rad }}+\frac{M(1-\lambda)}{\operatorname{Pr}} \frac{\mathrm{d} \overline{q_{t}}}{\mathrm{~d} z}\right] \delta(z)-\left[Q_{\text {rad }}+\frac{M(1-\lambda)}{\operatorname{Pr}} \frac{\mathrm{d} \overline{q_{t}}}{\mathrm{~d} z}\right] \frac{\mathrm{d} \delta(z)}{\mathrm{d} z} z_{s}
\end{aligned}
$$




\section{H. Fu}

$$
\begin{aligned}
& +\frac{M}{P r}\left(\left.\frac{\partial q_{t}^{\prime}}{\partial z}\right|_{z=0}-\left.\lambda \frac{\partial T^{\prime}}{\partial z}\right|_{z=0^{-}}\right) \delta(z) \\
\approx & \underbrace{-\frac{1-\gamma_{T}}{P r} \delta(z)}_{\text {basic state }}+\underbrace{\frac{1-\gamma_{T}}{P r} \frac{\mathrm{d} \delta(z)}{\mathrm{d} z} z_{s}}_{\text {interface undulation }}+\underbrace{\frac{M}{P r}\left(\left.\frac{\partial q_{t}^{\prime}}{\partial z}\right|_{z=0}-\left.\lambda \frac{\partial T^{\prime}}{\partial z}\right|_{z=0^{-}}\right) \delta(z)}_{\text {non-uniform evaporation }} .
\end{aligned}
$$

Here we have used (2.16) and (2.21). The quantities $\partial q_{l}^{\prime} /\left.\partial z\right|_{z=0^{-}}, \partial q_{t}^{\prime} /\left.\partial z\right|_{z=0}, \partial T^{\prime} /\left.\partial z\right|_{z=0^{-}}$ and $z_{s}$ all have small amplitude, so the product between them vanish in the linear analysis. On the right-hand side of (2.26), the first term denotes the basic state part, the second term denotes the interface undulation which produces a vertical dipole heating pattern, and the third term denotes the horizontally non-uniform evaporation which produces a monopole heating pattern. The basic state part leads to a piecewise linear $\bar{T}$ and, therefore, a piecewise constant $\mathrm{d} \bar{T} / \mathrm{d} z$, which corresponds to the $-w \mathrm{~d} \bar{T} / \mathrm{d} z=w\left[1-\left(1-\gamma_{T}\right) \mathcal{H}(z)\right]$ term in (2.23).

\section{Linear stability analysis}

The standard practice for treating a two-layer convection problem like this is to manipulate all the equations into a single high-order equation of $w$ for each layer respectively, and then find the proper matching condition (Ogura \& Kondo 1970). It is going to be shown that the moisture and radiative effects, which are the novel contributions of this work, are interfacial processes that only require a special configuration of the matching condition.

We call the case with the full non-uniform evaporation and interface undulation the 'free TRBC' (TRBC stands for two-layer Rayleigh-Bénard convection). For comparison, the classic TRBC where the cooling source is horizontally uniform and not undulating (Ogura \& Kondo 1970) is called the 'fixed TRBC'. Whenever we compare these two cases, the basic state temperature profile and, therefore, $\gamma_{T}$ is identical. The physics of the fixed TRBC reported by Whitehead \& Chen (1970) is briefly introduced here. The main body of the convective cell is constrained in the lower layer where there is a warm (cold) anomaly in the ascending (descending) region, so the vertical buoyancy flux is positive and kinetic energy is produced. In the upper layer the weak ascending (descending) flow generates a cold (warm) anomaly, so the vertical buoyancy flux is negative and the kinetic energy of the cell produced in the lower layer is partly diminished. The newly added radiation and evaporation should influence the instability with the buoyancy anomaly they induce. Whether such additional buoyancy anomalies favour the original cell or not should determine whether the new factors are stabilizing or destabilizing.

\subsection{The eigenvalue problem}

As the non-uniform evaporation and interface undulation take the form of a Dirac-delta function and its derivative in (2.23), their influence is concentrated near the saturation interface. Thus, compared with the fixed TRBC, the moisture effect only influences the matching condition. The procedure in this subsection is a standard practice that follows Ogura \& Kondo (1970), except the matching conditions of $\hat{T}$ and $D \hat{T}$ which are novel.

The normal mode solution is

$$
\begin{aligned}
\left\{u, v, w, p, T^{\prime}, q_{t}^{\prime}, q_{l}^{\prime}, z_{s}\right\}= & \left\{\hat{u}(z), \hat{v}(z), \hat{w}(z), \hat{p}(z), \hat{T}(z), \hat{q}_{t}(z), \hat{q}_{l}(z), \hat{z}_{s}\right\} \\
& \times \exp \left[\mathrm{i}\left(k_{x} x+k_{y} y\right)\right] \exp (\sigma t),
\end{aligned}
$$




\section{Saturation interface instability}

where $k_{x}$ is the $x$-direction wavenumber, $k_{y}$ is the $y$-direction wavenumber, $\sigma \equiv \sigma_{r}+\mathrm{i} \sigma_{i}$ is the complex growth factor, where $\sigma_{r}$ denotes the growth rate and $\sigma_{i}$ denotes the oscillation frequency. The z-dependent variables with a 'hat' denote the eigenfunctions. By substituting (3.1) into (2.18), (2.19) and (2.23), a sixth-order ordinary differential equation (ODE) of $\hat{w}$ is obtained:

$$
\left(\operatorname{Pr} \sigma+K^{2}-D^{2}\right)\left(\sigma+K^{2}-D^{2}\right)\left(K^{2}-D^{2}\right) \hat{w}= \begin{cases}\gamma_{T} \operatorname{Ra} K^{2} \hat{w}, & 0<z<\infty \\ \operatorname{Ra} K^{2} \hat{w}, & -1<z<0\end{cases}
$$

where $K^{2} \equiv k_{x}^{2}+k_{y}^{2}, D \equiv \mathrm{d} / \mathrm{d} z$. The derivative of the eigenfunctions will use notation $D$ rather than $\mathrm{d} / \mathrm{d} z$. At $z=-1$, the non-penetrative and free-slip velocity boundary condition, as well as the Dirichlet temperature boundary condition $\left.T^{\prime}\right|_{z=-1}=0$ that are adapted from (2.7) to yield

$$
\hat{w}=D^{2} \hat{w}=D^{4} \hat{w}=0, \quad z=-1 .
$$

As (3.2) have constant coefficients, obey the homogeneous lower boundary condition shown in (3.3) and the natural boundary condition at $z \rightarrow \infty$, we have

$$
\hat{w}(z)= \begin{cases}\sum_{m=4}^{6} a_{m} e^{-p_{m} z}, & 0<z<\infty, \\ \sum_{m=1}^{3} a_{m} \sinh \left[p_{m}(z+1)\right], & -1<z<0 .\end{cases}
$$

Here $a_{m}, m=1,2,3,4,5,6$ are the amplitude coefficients, $p_{m}$ are the eigenvalues. Substituting (3.4) into (3.2), and viewing $\sigma, R a$ and $K^{2}$ as coefficients, a complex cubic equation of $p_{m}^{2}$ is obtained and analytically solved using the method of Lebedev (1991). As the sign of $p_{m}$ is not constrained by the cubic equation, we choose the $p_{m}$ with a positive real part.

The six coefficients $a_{m}$ need to be determined by six matching conditions at $z=0$. The first four are the continuity of vertical velocity $w$, horizontal velocity $u$ and $v$, the non-dimensional tangential stress components $(\partial u / \partial z+\partial w / \partial x)$ and $(\partial v / \partial z+\partial w / \partial y)$, and pressure $p$. Using the normal mode form of (2.18) and (2.19), a few lines of algebra yield

$$
\left.D^{n} \hat{w}\right|_{z=0^{-}}=\left.D^{n} \hat{w}\right|_{z=0^{+}}, \quad n=0,1,2,3 .
$$

The remaining two conditions are about the disturbance temperature $T^{\prime}$ and heat flux $\operatorname{Pr}^{-1} \partial T^{\prime} / \partial z$. Unlike the fixed TRBC where $\hat{T}$ and $D \hat{T}$ are continuous at $z=0, \S \S 3.2$ and 3.3 will show that it is not the case for the free TRBC due to the interface undulation and the non-uniform evaporation there. The full solution procedure of the eigenvalue problem is shown in Appendix B.

\subsection{The effect of undulating interface}

The first term on the right-hand side of (2.23) denotes the interface undulation. First, we study how the small-amplitude interface displacement $\hat{z}_{s}$ produces a jump of $\hat{T}$ at $z=0$. Let $\epsilon$ be a small positive constant. The following double integral is performed on (2.23), within a small slot encompassing the interface. All terms vanish except the vertical 


\section{H. Fu}

diffusion term which has the highest order, and the $\mathrm{d} \delta(z) / \mathrm{d} z$ term which changes most abruptly:

$$
\begin{gathered}
\frac{1-\gamma_{T}}{P r} \hat{z}_{s} \int_{-\epsilon}^{\epsilon} \int_{-\epsilon}^{z^{\prime}} \frac{\mathrm{d} \delta(z)}{\mathrm{d} z} \mathrm{~d} z \mathrm{~d} z^{\prime} \sim-\frac{1}{P r} \int_{-\epsilon}^{\epsilon} \int_{-\epsilon}^{z^{\prime}} D^{2} \hat{T} \mathrm{~d} z \mathrm{~d} z^{\prime} \\
\Rightarrow \quad \frac{1-\gamma_{T}}{\operatorname{Pr}} \hat{z}_{s}+\frac{1}{\operatorname{Pr}}\left(\left.\hat{T}\right|_{z=0^{+}}-\left.\hat{T}\right|_{z=0^{-}}\right)=0 .
\end{gathered}
$$

Another more straightforward way to derive (3.6) is to use the interfacial temperature continuity $\left.T\right|_{z=z_{s}^{+}}=\left.T\right|_{z=z_{s}^{-}}$and perform Taylor expansion of $T$ near $z=0$, as has been employed by Hsieh (1972) in studying liquid-gas flow. The parameter $\operatorname{Pr}$ appears on both terms of the second line of (3.6) and can be eliminated.

Then, we consider the Clausius-Clapeyron equation that determines $z_{s}$. At the saturation interface, there is $q_{v s}=q_{t}$. The normal mode form of (2.20) yields

$$
\begin{gathered}
\left.\hat{q}_{t}\right|_{z=0}+\frac{\mathrm{d} \overline{q_{t}}}{\mathrm{~d} z} \hat{z}_{s}=\lambda\left(\left.\hat{T}\right|_{z=0^{-}}+\left.\frac{\mathrm{d} \bar{T}}{\mathrm{~d} z}\right|_{z=0^{-}} \hat{z}_{s}\right)=\lambda\left(\left.\hat{T}\right|_{z=0^{+}}+\left.\frac{\mathrm{d} \bar{T}}{\mathrm{~d} z}\right|_{z=0^{+}} \hat{z}_{s}\right) \\
\Rightarrow \quad \hat{z}_{s}=\frac{\left.\hat{q}_{t}\right|_{z=0}-\left.\lambda \hat{T}\right|_{z=0^{-}}}{1-\lambda}=\frac{\left.\hat{q}_{t}\right|_{z=0}-\left.\lambda \hat{T}\right|_{z=0^{+}}}{1-\lambda \gamma_{T}} .
\end{gathered}
$$

Fielder (1984) has derived an expression similar to (3.7) to find the cloud bottom undulation magnitude, where he considered the matching between a well-mixed sub-cloud layer and a well-mixed cloud layer.

The normal mode form of (2.18) and (2.19) are manipulated to represent $\hat{T}$ with $\hat{w}$ :

$$
\hat{T}=\frac{P r}{R a K^{2}}\left(D^{2}-K^{2}-\sigma\right)\left(D^{2}-K^{2}\right) \hat{w} .
$$

Substituting (3.7) and (3.8) into (3.6), we obtain the matching condition of $\hat{T}$ :

$$
\begin{aligned}
\left.\hat{T}\right|_{z=0^{+}} \frac{1-\lambda}{1-\lambda \gamma_{T}}-\left.\hat{T}\right|_{z=0^{-}}=\frac{\operatorname{Pr}}{\operatorname{RaK}^{2}}\left(D^{2}-K^{2}-\sigma\right)\left(D^{2}-K^{2}\right) \\
\times\left(\left.\hat{w}\right|_{z=0^{+}} \frac{1-\lambda}{1-\lambda \gamma_{T}}-\left.\hat{w}\right|_{z=0^{-}}\right)=\left.\underbrace{-\frac{1-\gamma_{T}}{1-\lambda \gamma_{T}}}_{\eta_{T}} \hat{q}_{t}\right|_{z=0},
\end{aligned}
$$

where $\eta_{T}$ is a negative constant. The $\left.\hat{w}\right|_{z=0^{+}}$and $\left.\hat{w}\right|_{z=0^{-}}$on the second line of the right-hand side denote the derivative value of $\hat{w}$ at $z=0^{+}$and $z=0^{-}$.

The quantity $\left.\hat{q}_{t}\right|_{z=0}$ is obtained by solving $q_{t}^{\prime}$ as an advective-diffusive tracer driven by $w$. The normal mode form of (2.22) is a second-order ODE of $\hat{q}_{t}$ :

$$
D^{2} \hat{q}_{t}-\left(K^{2}+\sigma P r\right) \hat{q}_{t}=-\hat{w} P r \quad \text { with }\left.\hat{q}_{t}\right|_{z=-1}=\left.\hat{q}_{t}\right|_{z \rightarrow \infty}=0 .
$$

It is solved with the method of variation of parameter in Appendix C. For $\sigma=0$, (3.10) can be viewed as the normal mode form of a Poisson equation with $-\hat{w} \operatorname{Pr}$ as the source term. Thus, when $\hat{w}$ is a real function (no phase tilt with height), $\hat{q}_{t}$ is real and has the same sign as $\hat{w}$. In other words, there is always positive $\left.q_{t}^{\prime}\right|_{z=0}$ at the updraft region for a neutral mode due to vertical advection.

The physical influence of the undulating interface is briefly analysed here, and illustrated in figure 2(a). The updraft at the saturation interface pushes the air at the 


\section{Saturation interface instability}

interface upward. These parcels are the coldest air in the vertical air column. The temperature field adjusts to the rising dome through diffusion, so the result is a cold anomaly at the upper layer and a warm anomaly at the lower layer, and vice versa in a valley produced by a downdraft. This produces a vertical dipole buoyancy anomaly, which corresponds to two undulation-induced torques which are in the opposite sign. A qualitatively similar yet more singular pattern can be produced by the interface undulation in a three-layer Rayleigh-Taylor instability model (Mellado et al. 2009). We note that the torque below the interface is in the same direction as the lower-layer convective cell (also a torque), which is generated by the unstable stratification. Because the main body of the convective cell is below the interface, the cell receives a more positive influence from the interfacial torque right below the interface than the negative influence from the torque right above the interface. Thus, the interface undulation is a destabilizing factor. A stricter explanation based on energetics is given in $\S 4.5$.

\subsection{The effect of non-uniform evaporation at the interface}

The second term on the right-hand side of (2.23) denotes the non-uniform liquid water diffusion and, therefore, evaporative cooling at the interface. It induces a jump of perturbation heat flux and, therefore, $D \hat{T}$ at $z=0$. This term is the key to reproduce the deformation-induced anomalous evaporative cooling at a 'flame front' (the interfacial ridge lifted by the updraft) described by Siems et al. (1990) and Mellado et al. (2009). The author is unaware of any previous work that addresses the influence of the deformation-induced anomalous evaporation on the interfacial instability.

Equation (2.23) indicates that the matching condition for heat flux in normal mode form is

$$
\left.D \hat{T}\right|_{z=0^{+}}-\left.D \hat{T}\right|_{z=0^{-}}=-M\left(\left.D \hat{q}_{t}\right|_{z=0}-\left.\lambda D \hat{T}\right|_{z=0^{-}}\right) .
$$

Substituting (3.8) into (3.11), we get the matching condition represented with $\hat{w}$ :

$$
\begin{aligned}
\frac{P r}{\operatorname{RaK^{2}}}\left(D^{2}-K^{2}-\sigma\right) & \left(D^{2}-K^{2}\right) D\left[\left.\hat{w}\right|_{z=0^{+}}-\left.(1+\lambda M) \hat{w}\right|_{z=0^{-}}\right] \\
= & -\left.M D \hat{q}_{t}\right|_{z=0} .
\end{aligned}
$$

As in $\S 3.2$, the $\left.\hat{w}\right|_{z=0^{+}}$and $\left.\hat{w}\right|_{z=0^{-}}$on the left-hand side denote the value of the derivatives of $\hat{w}$ at $z=0^{+}$and $z=0^{-}$. The expression of $\left.D \hat{q}_{t}\right|_{z=0}$ is documented in Appendix C.

In $\S 4$ the eigenfunctions of the neutral mode show that in most cases there is always $\partial q_{l}^{\prime} /\left.\partial z\right|_{z=0^{-}}<0$ at the updraft region $\left(\left.w\right|_{z=0}>0\right)$, primarily due to the squeezing of the $q_{t}$ contour surfaces near the interface. The detailed physical understanding and constraint are to be discussed in $\S 4.4$. Figure $2(b)$ illustrates the physical influence of non-uniform evaporation. There is more evaporative cooling in the updraft region than in the downdraft region, so the baroclinic torque produced by the non-uniform evaporation is opposite to the torque produced by the lower-level unstable stratification. Thus, the non-uniform evaporation is stabilizing. It is worth noting that $M$ only influences the instability via (3.11), not directly relevant to the interface undulation. Thus, a larger $M$ directly means a stronger non-uniform evaporation. 


\section{The physical mechanism}

\subsection{The investigation strategy}

Three reference tests of the linear stability problem are performed.

(i) The Ref-F test which is a fixed TRBC test that uses $\gamma_{T}=-2.5$ and $\operatorname{Pr}=1$. The matching condition uses $\left.\hat{T}\right|_{z=0^{+}}=\left.\hat{T}\right|_{z=0^{-}}$and $\left.D \hat{T}\right|_{z=0^{+}}=\left.D \hat{T}\right|_{z=0^{-}}$.

(ii) The Ref-E test with purely evaporative cooling that uses $M=6.364, \lambda=0.45, \gamma_{T}=$ -2.5 and $\operatorname{Pr}=1$ (corresponding to $Q_{\text {rad }} / \overline{Q_{\text {evap }}}=0$ ).

(iii) The Ref-ER test with both evaporative and radiative cooling that uses $M=3, \lambda=$ 0.45, $\gamma_{T}=-2.5$ and $\operatorname{Pr}=1$ (corresponding to $Q_{\text {rad }} / \overline{Q_{\text {evap }}}=1.12$ ).

Sensitivity tests are performed around Ref-ER, with an emphasis on studying the sensitivity to $Q_{\text {rad }} / \overline{Q_{\text {evap }}}$ by changing $\lambda$ and $\gamma_{T}$. The Ref-E test can be regarded as a special sensitivity test of the Ref-ER test with a larger $M$, which directly means a stronger non-uniform interfacial evaporation. Though this parameter set does not correspond to a specific state of real stratocumulus which is turbulent, it is an idealized state where the convective instability in the saturated layer, interfacial instability and upper-layer stratification all play a role. Only the marginal stability curve is studied in this section to understand the physical mechanism. Thus, $R a$ is to be solved, rather than prescribed. In $\S 5$ the weakly supercritical (linearly unstable) regime is briefly explored with nonlinear numerical simulation. The neutral mode might represent the large eddy component of a turbulent flow, with the small-scale eddies parameterized as the diffusivity (Zhou, Simon \& Chow 2014; Thuburn \& Efstathiou 2020). We leave the careful investigation of the relevance to the turbulent stratocumulus for future works.

The critical (marginally stable) $R a$ is defined as $R a_{c}$. The problem is horizontally isotropic, so there is no need to split the horizontal total wavenumber $K$ into $k_{x}$ and $k_{y}$. On a marginal curve, the minimum $R a_{c}$ is defined as $R a_{c m}$ and the corresponding $K$ is $K_{c m}$. For the single layer Rayleigh-Bénard convection at $\operatorname{Pr}=1$, the existence of minimum $R a_{c}$ can be heuristically understood as the competition between the destabilizing effect of the inviscid unstable gravity wave component, and the stabilizing effect of the diffusive and viscous component (Thuburn \& Efstathiou 2020). The supposed growth rate of the unstable gravity wave increases mildly with $K$ and asymptotically approaches $(R a / P r)^{1 / 2}$, which is the modulus of the non-dimensional imaginary Brunt-Väisälä frequency. The damping rate of diffusion increases as $K^{2}$. Thus, $R a_{c m}$ must be achieved at a finite $K=K_{c m}$. It will be shown that in this problem, a smaller $R a_{c m}$ is mostly accompanied with a smaller $K_{c m}$, similar to the fixed TRBC problem (Ogura \& Kondo 1970). Qualitatively speaking, a smaller $R a_{c m}$ means a smaller imaginary Brunt-Väisälä frequency, so less diffusion and therefore a lower $K_{c m}$ is needed to balance it.

By calculating the growth rate of various $R a$ in the parameter space around the reference test, we have not found any growing or neutral oscillatory mode $\left(\sigma_{r} \geq 0, \sigma_{i} \neq 0\right)$. Thus, we let the solver (introduced in Appendix B) only search across stationary modes $\left(\sigma_{i}=0\right)$ in all the tests. Whitehead (1968) has shown that an unstable or neutral oscillatory mode does not exist for the fixed TRBC problem (the principle of exchange of stability), but a rigorous proof is hard for our free TRBC problem. For the stationary mode, we arbitrarily set the phase by letting $\hat{w}$ be a real function with positive value in the lower layer. With this setting, the normal mode form of (2.18)-(2.23) show that all the eigenfunctions in (3.1) are real functions except $\hat{u}(z)$ and $\hat{v}(z)$. These real-value eigenfunctions are proportional to the physical space variables at an updraft column. 
(a)

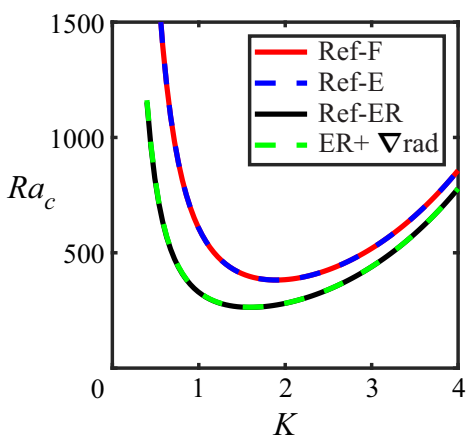

(b)

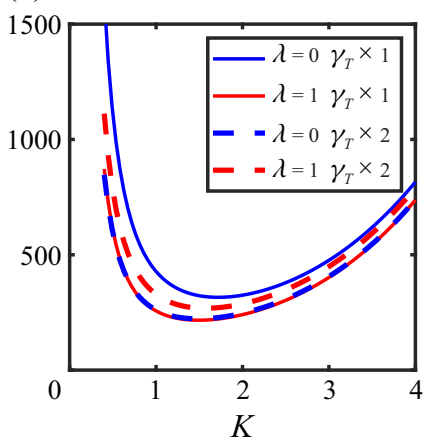

(c)

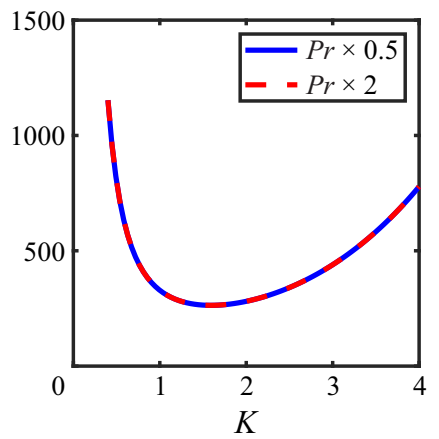

Figure 3. The marginal stability curve. (a) The solid red line is the Ref-F test, the dashed blue line is the Ref-E test, the solid black line is the Ref-ER test and the dashed green line is the Ref-ER test with the additional non-uniform radiative cooling at the interface (discussed in Appendix A). (b) The solid blue line and solid red line denote the $\lambda=0$ and $\lambda=1$ tests respectively for the reference $\gamma_{T}=-2.5$. The dashed blue line and dashed red line denote the corresponding tests for a double $\gamma_{T}$. In the code, we use $\lambda=10^{-10}$ to approximate $\lambda=0$ and $\lambda=1-10^{-10}$ to approximate $\lambda=1$, to avoid dividing by zero. (c) The solid blue line and dashed red line denote changing $\operatorname{Pr}$ to 0.5 and 2 times of their reference value $(\operatorname{Pr}=1)$, respectively. All tests in $(b)$ and $(c)$ are free TRBC tests with radiative cooling.

In Appendix D we introduce the novel finding that the purely evaporative cooling case yields an identical marginal stability curve (for the neutral mode) and growth rate (for the growing mode) to the corresponding fixed TRBC case (using the same $\gamma_{T}$ and $\operatorname{Pr}$ but for $\left.\hat{T}\right|_{z=0^{+}}=\left.\hat{T}\right|_{z=0^{-}}$and $\left.D \hat{T}\right|_{z=0^{+}}=\left.D \hat{T}\right|_{z=0^{-}}$). In $\S 4.5$ it is explained as the cancellation between the effect of the interface undulation and non-uniform evaporation with an argument of energetics.

\subsection{The lowest $R a_{c}$ neutral mode of the two reference tests}

Figure 3(a) shows the marginal stability curve of the Ref-F test, Ref-E test and Ref-ER test. The curve of the Ref-E test completely overlaps with that of the Ref-F test, which is strictly proved in Appendix D. The Ref-ER test has a lower $R a_{c m}$ and a smaller $K_{c m}$. We explain this more unstable behaviour as a smaller $M$ (larger $Q_{\text {rad }} / \overline{Q_{\text {evap }}}$ ) which makes the stabilizing effect of the non-uniform evaporation weaker than the destabilizing effect of the interface undulation.

The eigenfunctions and the reconstructed flow field in figure 4 show that, for all tests, there is a primary cell confined within the saturated layer. The Ref-E and Ref-ER tests have a less penetrative primary cell but a more prominent secondary upper cell than the Ref-F test, due to the baroclinic torque produced by the interface undulation. Though the Ref-E test and the Ref-F test have the same $R a_{c m}=381.82$ and $K_{c m}=1.90$, their eigenfunctions and therefore flow patterns are different. For the Ref-E and Ref-ER tests, the interface $z=z_{s}$ is raised at the updraft branch. Whether it is flatter than the $q_{t}$ contour lines is hard to see from this figure due to a 'technical issue' in the plotting: too large an amplitude violates the small-amplitude assumption, and too small an amplitude is hard to observe. This point will be carefully discussed in $\S 4.3$ and the interface will be shown to be no steeper. As there is $\left.D \hat{q}_{l}\right|_{z=0^{-}}<0$ in figure 4 for the Ref-E and Ref-ER tests, the perturbation liquid water gradient is negative at the updraft. This corresponds to a larger $q_{l}$ gradient at the updraft ridge of the interface, which produces more evaporation and explains the stabilizing effect of the non-uniform evaporation. 


\section{H. Fu}

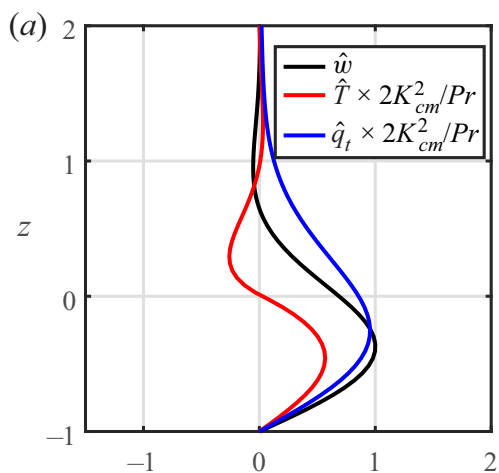

(b)

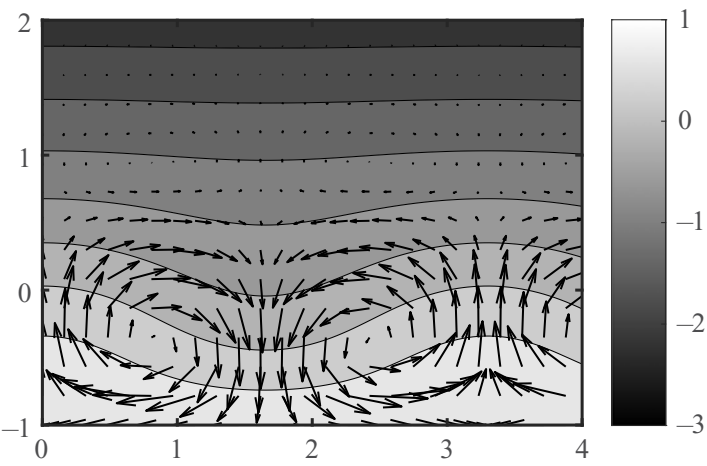

(c)

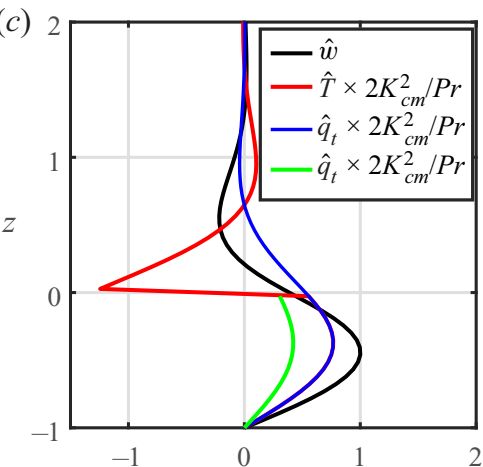

(e)

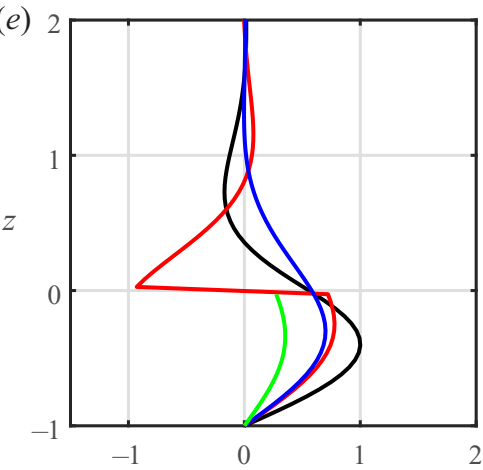

(d)

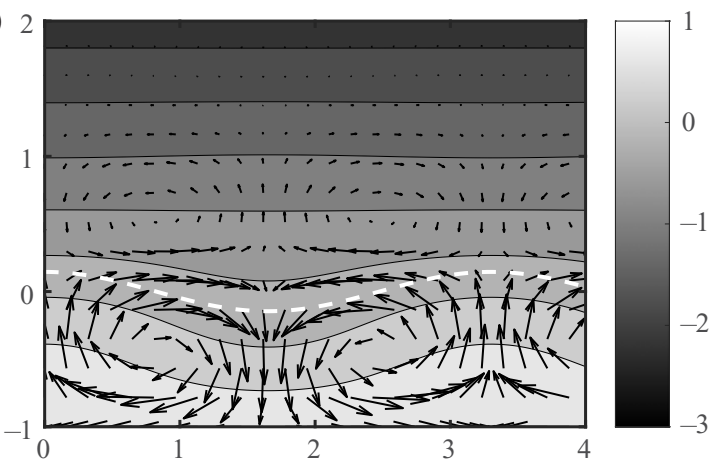

$(f)$

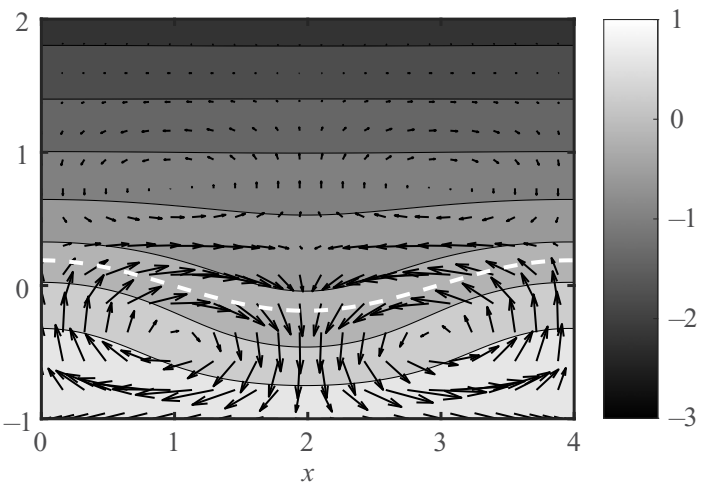

Figure 4. (a) The vertical profile of eigenfunction $\hat{w}$ (black line), $\hat{T}$ (red line) and $\hat{q}_{t}$ (blue line) for the Ref-F test. The magnitude is chosen by letting the maximum value of $\hat{w}$ be unit. The eigenfunctions $\hat{T}$ and $\hat{q}_{t}$ are further multiplied by $2 K_{c m}^{2} / P r$ to make their magnitude close to unit, because (2.22) and (2.23) are close to advection-diffusion equations. (b) The reconstructed 2-D $x-z$, flow field, with $k_{x}=K_{c m}$. The magnitude of $\hat{w}$ is set as $\max \{|\hat{w}|\}=2$. The velocity vector is shown as the arrows. The $q_{t}$ (as a passive tracer in the fixed TRBC) is shown as the contour lines, with each line corresponding to an increment of 0.4. The domain width ' 4 ' is arbitrary and does not mean a wavelength. (c) The same as (a), but for the Ref-E test, and with an additional $\hat{q}_{l}$ profile (green line) which is also multiplied by $2 K_{c m}^{2} / \operatorname{Pr}$. $(d)$ The same as $(b)$, but for the Ref-E test, and the additional dashed white line denotes the interface $z_{s}$. Plots $(e, f)$ are the same as $(c, d)$, but for the Ref-ER test where both evaporative and radiative cooling are present. Results are shown for (a) Ref-F $R a_{c m}=381.82 K_{c m}=1.90 ;(b)$ Ref-F $q_{t}$; (c) Ref-E $R a_{c m}=381.82 K_{c m}=1.90 ;(d)$ Ref-E $q_{t}$; (e) Ref-ER $R a_{c m}=263.46 K_{c m}=1.58 ;(f)$ Ref-ER $q_{t}$. 


\section{Saturation interface instability}

\subsection{The dynamics of interface undulation}

In the absence of radiative cooling $\left(Q_{\text {rad }} / \overline{Q_{\text {evap }}}=0\right)$ the interface has constant $T=0$ and $q_{t}=0\left(\hat{z}_{s}=\left.\hat{q}_{t}\right|_{z=0}\right.$ for any $\left.\lambda\right)$, a property due to the synchronous evolution of $q_{t}$ and enthalpy $h=\left(c_{p} T^{*}+L_{v} q_{v}^{*}-c_{p} T_{0}-L_{v} q_{v s 0}\right) /\left(c_{p} \Delta T\right)=T+M q_{v}$. This was pointed out in an equivalent thermodynamic framework by Mellado et al. (2009), and is derived with our thermodynamic framework in Appendix D. As $\left.\hat{q}_{t}\right|_{z=0}$ must be positive at the updraft, as has been discussed in $\S 3.2$, the saturation interface should rise at the updraft due to the moisture increment.

What is the physical mechanism for $T$ to be fixed in the $Q_{\text {rad }} / \overline{Q_{\text {evap }}}=0$ case? We explain it as a competition: for an updraft, the warming tendency due to the change of interface height is balanced by the cooling tendency due to the extra evaporative cooling at the interface. The warming tendency can be understood with an idealized purely diffusive problem of $T$, with the interface cooling rate fixed. Suppose $z_{s}$ moves upward at an updraft due to interface undulation. As the upper level is infinitely thick, the vertical thermal diffusion strongly adjusts the upper level temperature profile to the new diffusive-equilibrium profile, which makes $\left.T\right|_{z=z_{s}}$ rise. The full adjustment is impossible due to the damping by horizontal diffusion.

What if radiative cooling is considered? Equation (2.16) shows that the $Q_{\text {rad }} / \overline{Q_{\text {evap }}}$ is larger for a smaller $M$, larger $\lambda$ or larger $-\gamma_{T}$. The suppression of the instability by a larger $M$ has been discussed at the end of $\S 3.3$, so we focus on $\lambda$ and $\gamma_{T}$. We hypothesize that the addition of radiative cooling leads to a stronger interface undulation relative to the non-uniform evaporation, so the anomalous cooling at the updraft ridge of the interface is weaker compared with the warming tendency. Thus, the interfacial temperature $\left.T\right|_{z=z_{s}}$ should rise more above the basic state value $T=0$, and the Clausius-Clapeyron equation indicates that the saturation interface should fall behind the $q_{t}=0$ contour line $\left(\hat{z}_{s} /\left.\hat{q}_{t}\right|_{z=0}<1\right)$. The hypothesis is confirmed in figure $5(a)$, which shows that $\hat{z}_{s} /\left.\hat{q}_{t}\right|_{z=0}$ decreases as $\lambda$ or $-\gamma_{T}$ increases. In particular, there is $\hat{z}_{s}=\left.\hat{q}_{t}\right|_{z=0}$ for the $\lambda=0$ case, because the Clausius-Clapeyron equation (3.7) is independent of temperature when $\lambda=0$. The $\hat{z}_{s}=\left.\hat{q}_{t}\right|_{z=0}$ is a property shared by the $Q_{\text {rad }} / \overline{Q_{\text {evap }}}=0$ (yet $\lambda \neq 0$ ) case. The analysis leads to an inference: even though radiative cooling enhances interface undulation, the decrease of $\hat{z}_{s} /\left.\hat{q}_{t}\right|_{z=0}$ means that it is self-limiting.

In fact, a quantitative lower bound of $\hat{z}_{s} /\left.\hat{q}_{t}\right|_{z=0}$ can be found. Note that $\hat{z}_{s}$ can be expressed with either $\hat{T}_{z=0^{-}}$or $\hat{T}_{z=0^{+}}$in (3.7). As long as $\hat{z}_{S}$ is positive (the interface rises at the updraft), both the movement of the interfacial cooling source and the direct vertical advection of the basic state temperature make $\hat{T}_{z=0^{-}} \gtrsim 0$ and $\hat{T}_{z=0^{+}} \lesssim 0$. Substituting this argument into (3.7), and combining it with the physical argument $\left.\hat{z}_{s} \lesssim \hat{q}_{t}\right|_{z=0}$, we obtain a range estimation of $\hat{z}_{s}$ :

$$
\left.\frac{\left.\hat{q}_{t}\right|_{z=0}}{1-\lambda \gamma_{T}} \lesssim \hat{z}_{s} \lesssim \hat{q}_{t}\right|_{z=0} .
$$

The lower bound is plotted as the dashed lines in figure 5(a). Though this constraint is loose, the lower bound does grasp the decrease of $\hat{z}_{s}$ with $\lambda$, as well as a faster decrease for a higher $-\gamma_{T}$ (with fixed $M$ and $P r$ ).

\subsection{The liquid water gradient near the interface}

Equation (2.23) shows that the sign of $\partial q_{l}^{\prime} / \partial z$ near the interface depends on $\partial q_{t}^{\prime} / \partial z$ and $\partial T^{\prime} / \partial z$. As the basic state $q_{t}$ and $T$ in the lower layer are both decreasing with height, there is a positive $q_{t}$ and $T$ anomaly at the updraft region. The flow is horizontally divergent as 


\section{H. Fu}
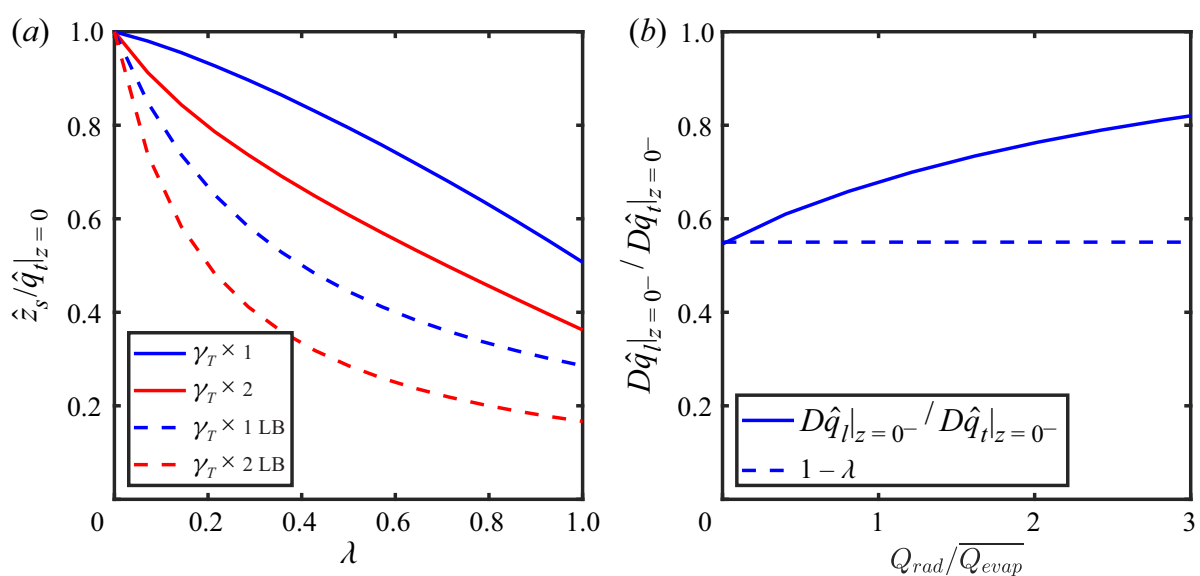

Figure 5. (a) The change of $\hat{z}_{s} /\left.\hat{q}_{t}\right|_{z=0}$ with $\lambda$ for two $\gamma_{T}$ values, with other parameters identical to the Ref-ER test. The solid blue line denotes the test using the reference value $\gamma_{T}=-2.5$, and the solid red line denotes the double $\gamma_{T}$ test. The dashed blue and dashed red lines denote $\left(1-\lambda \gamma_{T}\right)^{-1}$ for the normal and double $\gamma_{T}$ test, respectively, which are the theoretical estimates of the lower bound (abbreviated as LB). (b) The dependence of $\left.D \hat{q}_{l}\right|_{z=0^{-}} /\left.D \hat{q}_{t}\right|_{z=0}$ on $Q_{\text {rad }} / \overline{Q_{\text {evap }}}$ (solid blue line), performed by changing $\gamma_{T}$ while fixing $\lambda$ and $M$ to the Ref-ER test value. The dashed blue line is $(1-\lambda)$ which is an estimated lower bound of $\left.D \hat{q}_{l}\right|_{z=0^{-}} /\left.D \hat{q}_{t}\right|_{z=0}$.

the updraft intersects the interface, so the $q_{t}$ contour surfaces are squeezed and there is a negative $\partial q_{t}^{\prime} / \partial z$, as has been discussed in $\S 3.3$. The rising of the interface cooling source also induces a warming anomaly right below the interface (e.g. figure $2 a$ ), which locates above the peak warming anomaly induced by the upward advection of the unstable basic state. Thus, the negative $\left.D \hat{T}\right|_{z=0^{-}}$should rise towards zero, as can be seen by comparing figures $4(c)$ and $4(e)$.

This can be partly quantified. We start from the $Q_{\text {rad }} / \overline{Q_{\text {evap }}}=0$ case introduced in Appendix D, where (D7) shows $\hat{q}_{t}=\hat{T}$ for $z<0$. This indicates

$$
\left.D \hat{q}_{l}\right|_{z=0^{-}}=\left.(1-\lambda) D \hat{q}_{t}\right|_{z=0} \text { for } Q_{\text {rad }} / \overline{Q_{\text {evap }}}=0 .
$$

When radiative cooling is incorporated and gradually raised by increasing $-\gamma_{T}$, the $\hat{T}$ jump at $z=0$ should increase according to (3.6). Thus, we expect that as $-\gamma_{T}$ increases, $\left.D \hat{q}_{l}\right|_{z=0^{-}}$will approach $\left.D \hat{q}_{t}\right|_{z=0}$. This argument and (4.2) lead to a constraint of $\left.D \hat{q}_{l}\right|_{z=0^{-}} /\left.D \hat{q}_{t}\right|_{z=0}$ :

$$
1-\lambda \lesssim \frac{\left.D \hat{q}_{l}\right|_{z=0^{-}}}{\left.D \hat{q}_{t}\right|_{z=0}} \lesssim 1 .
$$

For a $-\gamma_{T}$ as large as 20 (very strong interfacial undulation), $\left.D \hat{T}\right|_{z=0^{-}}$only marginally exceeds 0 and $\left.D \hat{q}_{l}\right|_{z=0^{-}} /\left.D \hat{q}_{t}\right|_{z=0}$ marginally exceeds 1 . We have not figured out the mechanism of this asymptotic behaviour. Equation (4.3) is verified in figure $5(b)$ which shows that $\left.D \hat{q}_{l}\right|_{z=0^{-}} /\left.D \hat{q}_{t}\right|_{z=0}$ does increase mildly with increasing $Q_{\text {rad }} / \overline{Q_{\text {evap }}}$ (performed by changing $\gamma_{T}$ and fixing $\lambda$ ), with $(1-\lambda)$ as its lower bound. This indicates a coupling of the two effects: a stronger interface undulation tends to enhance the non-uniform evaporation to some extent. Unfortunately, (4.3) is not accurate enough to include $\gamma_{T}$.

With (4.1) and (4.3) in hand, we try to answer whether $\lambda$ is a destabilizing factor or not. First, we look at the marginal curve of different $\lambda$ for fixed $\gamma_{T}$. Figure $3(b)$ shows that for the reference value $\gamma_{T}=-2.5$, a larger $\lambda$ significantly destabilizes the system. For double $\gamma_{T}$, however, a larger $\lambda$ weakly stabilizes the system. This indicates that a larger 


\section{Saturation interface instability}

$-\gamma_{T}$ (stronger upper-layer stratification) gradually shifts $\lambda$ from a destabilizing factor to a stabilizing one. Physically, this is due to two competing factors. For given $-\gamma_{T}$, a larger $\lambda$ suppresses $\hat{z}_{s}$ and therefore weakens the interface undulation as shown in (4.1), but it also weakens the non-uniform evaporation as shown in (4.3) due to a smaller magnitude of liquid water gradient. The dependence of the relative strength of the two factors on $\gamma_{T}$ is not quantitatively constrained at present.

\subsection{The relative importance of interface undulation and non-uniform evaporation}

The relative strength of the destabilizing effect of the undulating interface and the stabilizing effect of non-uniform evaporation is quantified by comparing their relative contribution to the tendency of the vertical buoyancy flux $\overline{w T^{\prime}}$. Here the overline on perturbation quantities denotes horizontal average in a wavelength. Multiplying $w$ to (2.23), multiplying $T^{\prime}$ to the $w$ component of (2.19), and then summing them up and performing a horizontal wavelength average, we get

$$
\begin{gathered}
\frac{\partial \overline{w T^{\prime}}}{\partial t}=\overline{w\left(\frac{\partial T}{\partial t}\right)_{I U}}+\overline{w\left(\frac{\partial T}{\partial t}\right)_{N E}}+\text { etc. } \\
\overline{w\left(\frac{\partial T}{\partial t}\right)_{I U}} \equiv \hat{w} \frac{1-\gamma_{T}}{P r} \hat{z}_{S} \frac{\mathrm{d} \delta(z)}{\mathrm{d} z},\left.\quad \overline{w\left(\frac{\partial T}{\partial t}\right)_{N E}} \equiv \hat{w} \frac{M}{\operatorname{Pr}} D \hat{q}_{l}\right|_{z=0^{-}} \delta(z),
\end{gathered}
$$

where 'IU' denotes the contribution from interface undulation and 'NE' denotes that from non-uniform evaporation. Other terms on the right-hand side of the buoyancy flux tendency equation (4.4), which do not involve the interfacial process, are not shown. The relative importance of the two mechanisms can be roughly estimated as the ratio of the vertical integral of $\overline{w(\partial T / \partial t)_{I U}}$ to $\overline{w(\partial T / \partial t)_{N E}}$. As their contributions are opposite in sign, their ratio with a minus sign is defined as a positive quantity $\Phi$,

$$
\Phi \equiv-\frac{\int_{-1}^{\infty} \overline{w\left(\frac{\partial T}{\partial t}\right)_{I U}} \mathrm{~d} z}{\int_{-1}^{\infty} \overline{w\left(\frac{\partial T}{\partial t}\right)_{N E}} \mathrm{~d} z}=-\frac{\int_{-1}^{\infty} \hat{w} \frac{1-\gamma_{T}}{P r} \hat{z}_{S} \frac{\mathrm{d} \delta(z)}{\mathrm{d} z} \mathrm{~d} z}{\left.\int_{-1}^{\infty} \hat{w} \frac{M}{\operatorname{Pr}} D \hat{q}_{l}\right|_{z=0^{-}} \delta(z) \mathrm{d} z}=\frac{\left.\frac{1-\gamma_{T}}{\operatorname{Pr}} \hat{z}_{S} D \hat{w}\right|_{z=0}}{\left.\left.\frac{M}{\operatorname{Pr}} \hat{w}\right|_{z=0} D \hat{q}_{l}\right|_{z=0^{-}}} .
$$

To make the undulating interface render a positive buoyancy flux tendency and favour the convective cell, the interface must locate at the upper part of the convective cell $\left(\left.D \hat{w}\right|_{z=0}<\right.$ 0 ), so that the $\hat{w}$ at the heating (lower) part of the interfacial dipole is larger than that at the cooling (upper) part, as shown in (4.6) and illustrated in figure 2(a).

Substituting the estimate of $\hat{z}_{S}$ (4.1) and that of $\left.D \hat{q}_{l}\right|_{z=0^{-}}$(4.3) into (4.6), we get

$$
\frac{1}{M} \frac{1-\gamma_{T}}{1-\lambda \gamma_{T}} \frac{\left.\left.\hat{q}_{t}\right|_{z=0} D \hat{w}\right|_{z=0}}{\left.\left.\hat{w}\right|_{z=0} D \hat{q}_{t}\right|_{z=0}} \lesssim \Phi \lesssim \frac{1}{M} \frac{1-\gamma_{T}}{1-\lambda} \frac{\left.\left.\hat{q}_{t}\right|_{z=0} D \hat{w}\right|_{z=0}}{\left.\left.\hat{w}\right|_{z=0} D \hat{q}_{t}\right|_{z=0}} .
$$

Figure 4 shows that the eigenfunctions of $\hat{w}$ and $\hat{q}_{t}$ have a similar shape, so there is $\left(\left.\left.\hat{q}_{t}\right|_{z=0} D \hat{w}\right|_{z=0}\right) /\left(\left.\left.\hat{w}\right|_{z=0} D \hat{q}_{t}\right|_{z=0}\right) \sim 1$. With this, we use (2.16) to rewrite (4.7), and get

$$
\left(1+\frac{Q_{\text {rad }}}{\overline{Q_{\text {evap }}}}\right) \frac{1-\lambda}{1-\lambda \gamma_{T}} \lesssim \Phi \lesssim 1+\frac{Q_{\text {rad }}}{\overline{Q_{\text {evap }}}} .
$$




\section{H. Fu}

The inequalities (4.7) and (4.8) indicate the following.

(i) The destabilizing effect of the undulating interface can be stronger than the stabilizing effect of non-uniform evaporation only when the radiative cooling is present.

(ii) The upper bound of the net destabilizing effect is roughly proportional to the ratio of the basic state interfacial radiative cooling rate to the diffusion-induced evaporative cooling rate. It confirms that raising $Q_{\text {rad }} / \overline{Q_{\text {evap }}}$ by decreasing $M$ (fixing $\lambda$ and $\gamma_{T}$ ) makes the system more unstable.

(iii) The energetics argument is unable to depict the change of the instability behaviour on $\lambda$ for different $\gamma_{T}$ (e.g.by calculating $\partial^{2} \Phi /\left(\partial \lambda \partial \gamma_{T}\right)$ ), which is discussed in $\S 4.4$. This is due to a lack of the way to include $\gamma_{T}$ into the inequality (4.3).

Another invariant property is that changing $\operatorname{Pr}$ alone does not influence the marginal curve and the eigenfunctions at all. The former is illustrated in figure $3(c)$. Both properties are evident by checking the sixth-order ODE in (3.2) and the matching conditions in (B1), or the heuristic comparison in (4.7). To explain this, first we note that $\operatorname{Pr}$ itself does not influence the neutral mode of the fixed TRBC where $v$ and $\kappa$ are equivalent dissipating factors. Second, we note that $\operatorname{Pr}$ does not influence the moist process. This originates from the assumption of identical thermal and water diffusivity, which makes the interfacial moist processes detached from $\operatorname{Pr}$. For $R a>R a_{c}, \operatorname{Pr}$ still has an influence on the growth rate, because the time evolution of the $\hat{q}_{t}$ (3.10) involves $P r$.

\section{Nonlinear numerical simulation}

In this section fully nonlinear numerical simulations of the non-dimensional version of (2.1)-(2.6) are used to validate the linear stability analysis, and demonstrate the flow pattern in the weakly nonlinear regime.

The simulation code is built by the author using a standard two-dimensional Fourier spectral method (Durran 2010). The governing equation is in vorticity-stream function formulation, coupled to an equation of $q_{t}$ and an equation of enthalpy $h=T+M q_{v}$. The $h$ equation is

$$
\frac{\partial h}{\partial t}+\boldsymbol{u} \cdot \nabla h=\kappa \nabla^{2} h+Q_{r a d} \delta\left(z-z_{s}\right),
$$

whose only source term is radiative cooling. In the code $Q_{\mathrm{rad}} \delta\left(z-z_{s}\right)$ is regularized to $Q_{\mathrm{rad}} /\left(\pi^{1 / 2} l_{\text {rad }}\right) \exp \left[-\left(z-z_{s}\right)^{2} / l_{\text {rad }}^{2}\right]$, where $l_{\text {rad }}=0.05$ is a fixed regularization length scale. Using (2.4), the temperature $T$ is diagnosed from $q_{t}$ and $h$ with a maximum operator, which is equivalent to the 'dry and moist buoyancy' formulation of Bretherton (1987):

$$
T=\max \left\{\frac{h}{1+\lambda M}, h-q_{t} M\right\} .
$$

The position where the two quantities equal each other is $z_{s}$. This formulation avoids the direct treatment of evaporative cooling, so it is convenient for numerical simulation. Imposing a material derivative on (5.2) and then expressing the maximum operator with Heaviside function yields the non-dimensional version of (2.6), which has explicit evaporative cooling and is therefore more suitable for theoretical analysis. A note of the derivation is deposited in the supplemental material available at https://doi.org/10.1017/ jfm.2021.784. In the code, the $\alpha$-softmax function is used as a smoothed maximum operator: $S_{\alpha}\left(\phi_{1}, \phi_{2}\right)=\left(\phi_{1} e^{\alpha \phi_{1}}+\phi_{2} e^{\alpha \phi_{2}}\right) /\left(e^{\alpha \phi_{1}}+e^{\alpha \phi_{2}}\right)$ (Lange et al. 2014), where $\phi_{1}$ 

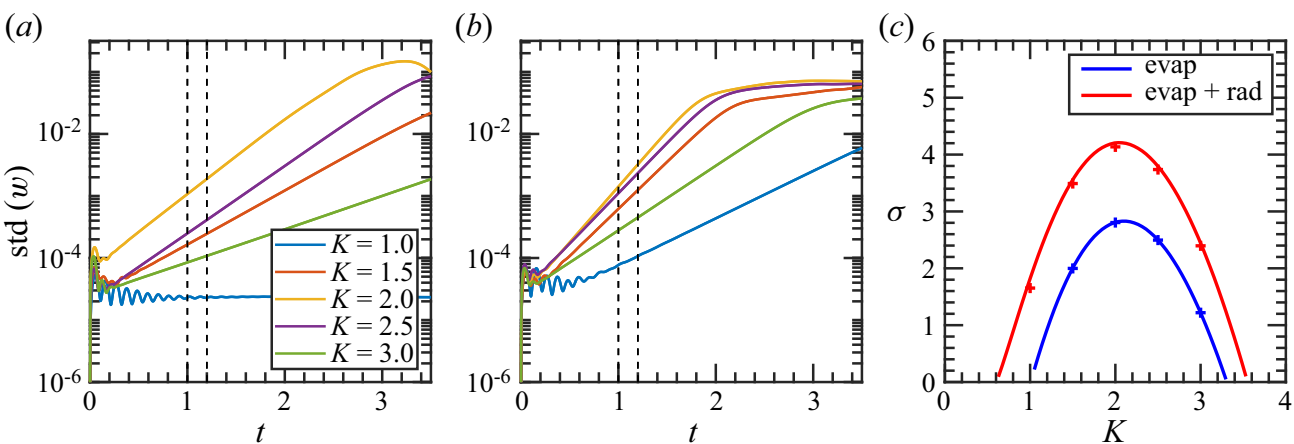

Figure 6. (a) The time series of the standard deviation of $w$, denoted as $\operatorname{std}(w)$, for $K=1.0$ (blue line), $K=1.5$ (red line), $K=2.0$ (yellow line), $K=2.5$ (purple line), $K=3.0$ (green line) tests of the nonlinear numerical simulation, for the Growth-E tests. The two dashed black lines denote $t_{1}=1.0$ and $t_{2}=1.2$ at which the $\operatorname{std}(w)$ is sampled to calculate the growth rate $\sigma=\left\{\ln \left[\left.\operatorname{std}(w)\right|_{t_{2}}\right]-\ln \left[\left.\operatorname{std}(w)\right|_{t_{1}}\right]\right\} /\left(t_{2}-t_{1}\right)$. (b) The same as (a), but for the Growth-ER tests. (c) The growth rate against wavenumber $K$. The theoretical calculation for the Growth-E tests is shown as the solid blue line, and that diagnosed from the nonlinear numerical simulation is shown as the blue '+.' Those corresponding to the Growth-ER tests are shown as the solid red line and red '+.' The Growth-E test at $K=1$ has a negative growth rate (very close to zero) and does not appear in this $\sigma>0$ plotting.

and $\phi_{2}$ are arbitrary input variables. The variable $z_{s}$, which must be accurately determined to calculate the radiative cooling term, is set by vertically interpolating $\phi_{1}$ and $\phi_{2}$ on a very fine mesh, and then using the unmodified maximum operator to find the zero-crossing point of $\phi_{1}-\phi_{2}$. We choose $\alpha=20$, which leads to a saturation interface thickness of about $\alpha^{-1}=0.05$ for $O\left(\phi_{1}-\phi_{2}\right) \sim 1$. The second-order Adams-Bashforth scheme is used in time stepping. Zero padding is used to accurately calculate the product terms of all resolved waves in the physical space. No hyperdiffusion is used.

The infinite upper boundary in the linear stability analysis is replaced by a rigid lid in the simulation, which is located at a distance $l$ above the basic state interface $z=0$. The length $l$ must be much larger than the convective penetration depth $H_{p}=-\gamma_{T}^{-1}$ to make the upper lid a good approximation of the infinite boundary. As a modification from (2.8), the upper lid has free-slip velocity, fixed $h$ and $q_{t}$,

$$
\left.h\right|_{z=l}=-\gamma_{T} l-M l,\left.\quad q_{t}\right|_{z=l}=-l .
$$

The parameter set is identical to the reference test shown in $\S 4.1$, except that we use a supercritical $R a=600$ to study the unstable mode. The domain depth in the $z$-direction is $1+l$, with $l=3$. The domain width in the $x$-direction is different from test to test. The vertical grid point number is 256, equivalent to a vertical grid interval of $\Delta z=0.0156$. This guarantees at least three grid points in the radiative cooling slot whose thickness is $l_{\text {rad }}$, and also three points in the saturation interface whose thickness is $\alpha^{-1}$. The horizontal grid point number is 4 for the ten small-amplitude growth rate benchmark tests, and 64 for the two flow pattern tests which extend to the finite-amplitude regime. The time step is $\Delta t=0.041(\Delta z)^{2}$. The initial condition is the analytical solution of radiative-diffusive equilibrium (running for 0.1 time units with the advection term off), plus a smoothed noise on $h$.

Among the ten low-horizontal resolution simulations that aim to benchmark the growth rate, the first five runs, named Growth-E tests, are for the purely evaporative cooling case. They have the same parameter as the Ref-E test except for the given $R a$. The horizontal domain width takes $L_{x}=2 \pi / K$, where $K=1.0,1.5,2.0,2.5$ and 3.0. 
(a)
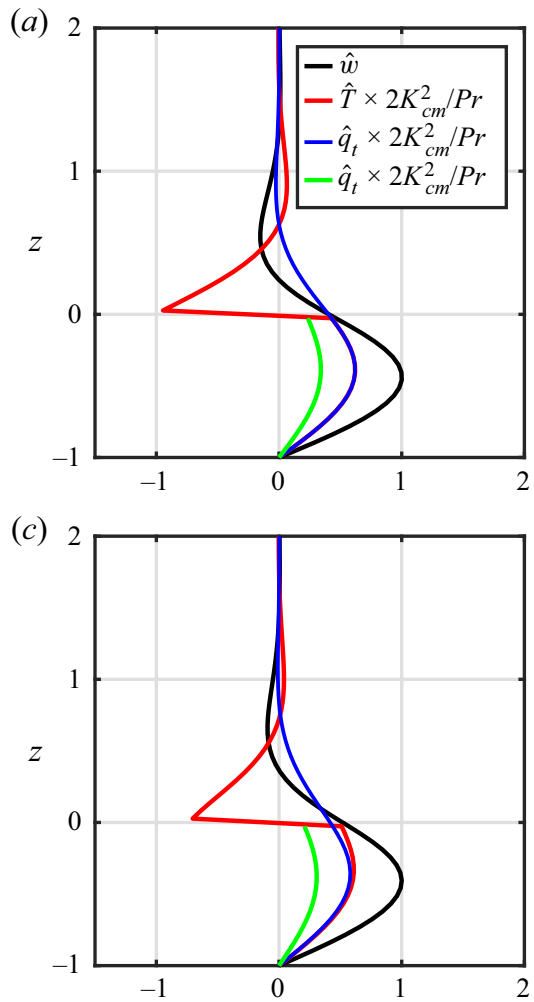

(b)

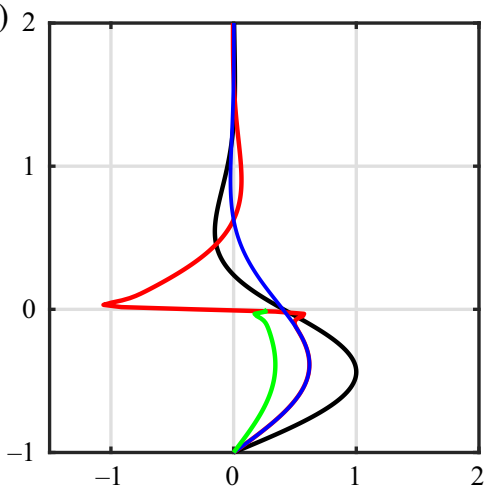

(d) 2

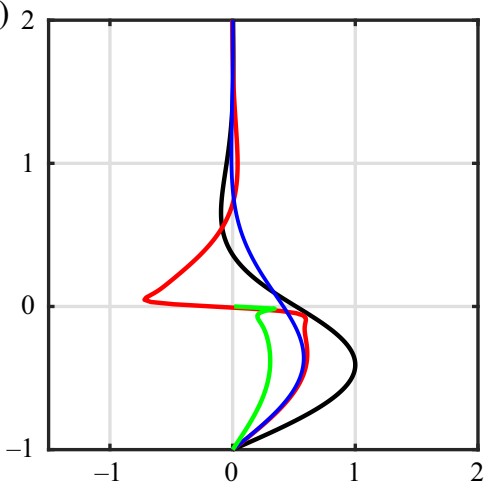

Figure 7. (a) The theoretical eigenfunctions for the Growth-E parameter test with $K=2.0$. (b) The normalized vertical profiles at the maximum $w$ column of the high-resolution numerical simulation that uses the Growth-E parameter. The profiles are calculated by subtracting the horizontal average, which are very close to the piecewise linear $\bar{T}$ in the small-amplitude regime. The labels and the normalization procedure are identical to those in figure 4 . The simulation data uses the $t=1.4$ snapshot. Plots $(c, d)$ are the same as $(a, b)$, but for the Growth-ER parameter test. The $\hat{q}_{l}$ anomaly near $z=0$ in $(b, d)$ is due to the smoothing treatment of (5.2) in the numerical model. Results are shown for $(a)$ evap, theory; $(b)$ evap, simulation; $(c)$ evap + rad, theory; (d) evap + rad, simulation.

The second five runs, named Growth-ER tests, are for the mixed evaporative-radiative cooling tests which use the same array of $K$. The parameters are the same as the Ref-ER test except for the given $R a$. The time series of the standard deviation of $w$ are used as the norm for calculating the growth rate, as shown in figure $6(a, b)$. An exponentially growing range of the stationary mode is clear for each run, though there is some initial oscillation which is probably due to the decaying oscillatory mode. The growth rate of all simulations match well with the theoretical prediction of the horizontally longest wave, as shown in figure $6(c)$. The Growth-ER tests grow faster than the Growth-E tests, which is not surprising given that the Ref-ER test has a lower $R a_{c m}$ than the Ref-E test.

Two high-resolution tests with 64 grid points in the $x$-direction are performed for the Growth-E and Growth-ER parameters. Both tests have a domain width of $L_{x}=2 \pi / K$, with $K=2.0$ which is close to the most unstable wavenumber of each test (e.g.figure $6 c$ ). Their vertical profiles at $t=1.4$ match well with the respective theoretical eigenfunctions of the $R a=600$ and $K=2.0$ modes, as shown in figure 7. The result is not sensitive to the choice of $t$, so long as it is in the small-amplitude regime. Figure 8 shows that in the finite-amplitude regime of both tests, the cold air produced at the ridge of the interface 

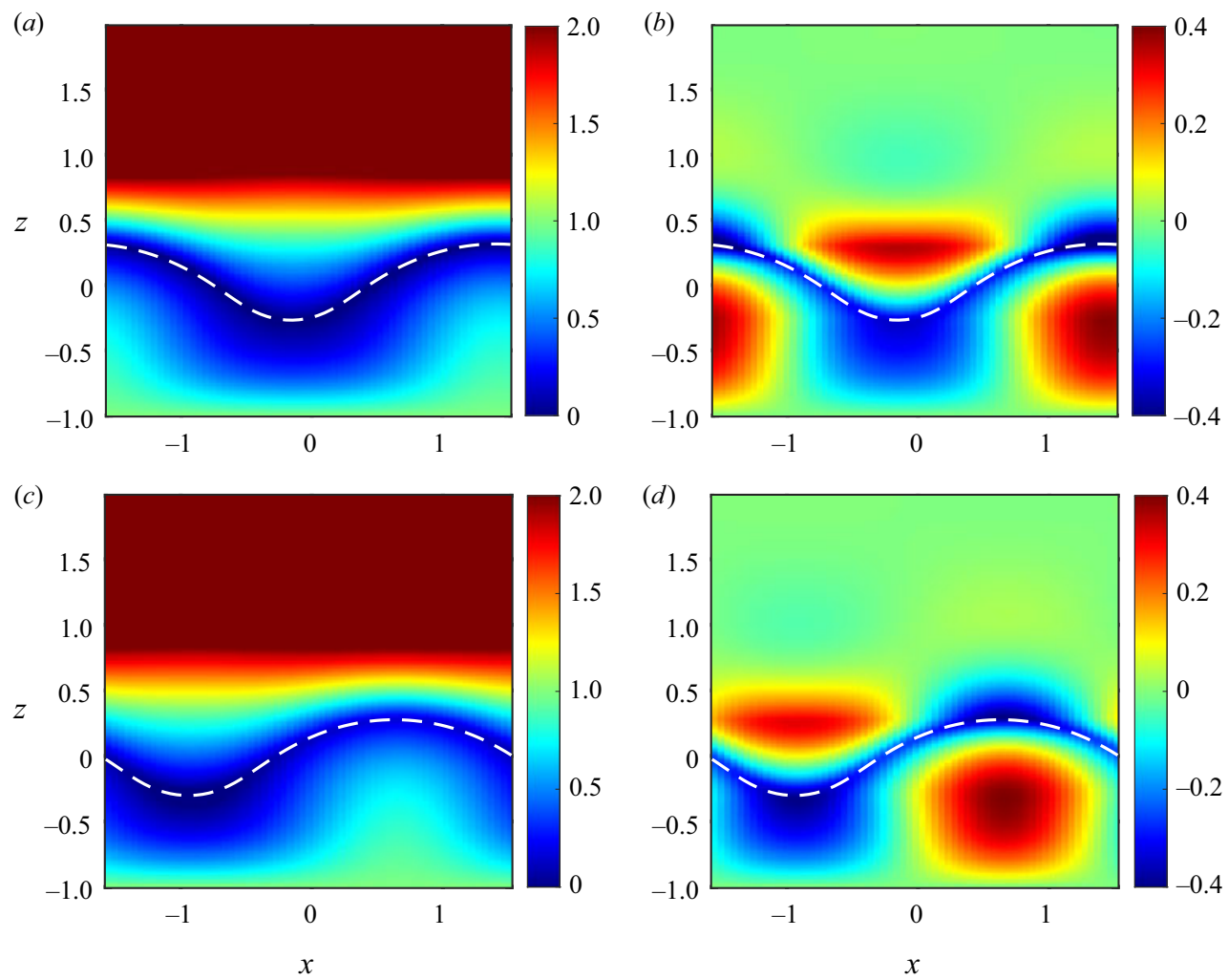

Figure 8. The $T$ and $T^{\prime}$ (calculated by subtracting the horizontal average profile which is smoother than $\bar{T}$ in this finite-amplitude regime) of the Growth-E parameter test high-resolution simulation at $t=3.2$ are shown in $(a, b)$, respectively. Plots $(c, d)$ are the same as $(a, b)$, but for the Growth-ER parameter test and a time snapshot at $t=2.3$. The motivation of using two different time snapshots is to make their amplitude comparable. The white dashed line denotes the saturation interface. Only the $-1 \leq z \leq 2$ part of the domain is shown, because the upper level has little deviation from the basic state. Results are shown for $(a) t=3.20$ evap $T$; $(b) t=3.20$ evap $T^{\prime}$; (c) $t=2.30$ evap $+\operatorname{rad} T ;(d) t=2.30$ evap $+\operatorname{rad} T^{\prime}$.

(the 'flame front') falls towards the valley of the interface. This is similar to that reported in the local staircase initial state simulations of Siems et al. (1990) and Mellado et al. (2009), though the structure here is smoother due to the smoother basic state profile. The radiative cooling influences the growth rate in this framework, but it does not change the flow pattern dramatically.

In the nonlinear regime the moist process is no longer the simple balance between the interface undulation and the non-uniform evaporation. As suggested by one of the reviewers, a detailed comparison between the fixed TRBC and the free TRBC in the weakly nonlinear regime can be made. For the turbulent regime, the moist convection has the distinctive feature that the vortex ring associated with the 'flame front' can engulf the upper-layer air and produce further turbulence through evaporative cooling (Siems et al. 1990). However, in the turbulent regime the strong horizontal mixing might homogenize the horizontal cooling difference, as suggested by de Lozar \& Mellado (2013). It is unclear whether the two mechanisms here are still important for the large eddy dynamics of the turbulent cloud. 


\section{H. Fu}

\section{Conclusion}

We present a novel two-layer convective instability problem, with the lower layer thermally unstable and saturated to water vapour, and the upper layer stable and unsaturated. This is a prototype model for understanding the cloud or fog top mixing. Two novel mechanisms which compete with each other are found.

First, the undulation of the interface is a destabilizing effect. The interface as a radiative and evaporative cooling source induces a vertical dipole buoyancy anomaly, which is superposed on the upper part of the lower-layer convective cell and therefore favours further undulation.

Second, the non-uniform evaporation at the interface is a stabilizing effect. There is stronger interfacial evaporative cooling at the updraft region due to the larger $q_{l}$ (liquid water) gradient magnitude near the interface, and vice versa at the downdraft. At the updraft, this is induced by a larger $q_{t}$ gradient due to the squeezing of $q_{t}$ contour surfaces by the horizontally divergence flow of the lower-layer primary cell, as well as a more complicated temperature gradient adjustment.

The competition between the destabilizing effect of undulating interface and the stabilizing effect of non-uniform evaporation is quantified by calculating the ratio of their contribution to the tendency of the vertical buoyancy flux. Without radiative cooling, the two effects break even. With radiative cooling, $Q_{\text {rad }} / \overline{Q_{\text {evap }}}$ can roughly measure the net destabilizing effect of radiative cooling, which enhances the interface undulation but does not directly influence the non-uniform evaporation.

As for the flow pattern, the baroclinic torque generated near the interface makes the primary convective cell more confined in the lower layer and favours the secondary cell in the upper layer. The squeezed $q_{t}$ contour surfaces at the interfacial ridge can be viewed as a quantification of the 'flame front' structure seen in the cloud-top mixing simulation (Siems et al. 1990; Mellado et al. 2009), though the front is smoother due to the smoother basic state profile in this theory. The subsequent aggregation of cold air in the valley of the interface is grasped in the nonlinear simulation.

This research is far from complete. Future works may include the following.

(i) The proof of principle of exchange of stability for the case with non-zero radiative cooling, if it exists.

(ii) Using numerical simulation to investigate the instability with initial state profiles that lie between the local staircase buoyancy profile of Siems et al. (1990) and our global piecewise linear one. It may better link the real cloud-top mixing problem and this simple model. An investigation of the weakly nonlinear regime, as has been done by Ogura \& Yagihashi (1971) for the fixed TRBC, is a necessary intermediate step to link our linear theory to the turbulent regime.

(iii) An extension to consider the influence of vapour and liquid water on buoyancy, as well as the droplet settling. The first two factors can be readily included into the current framework. Their coupling to droplet settling is a challenging topic.

(iv) An extension to study the instability of the cloud bottom (e.g.mammatus cloud formation), which is a saturation interface that is susceptible to evaporative cooling and the heating of the surface-emitted longwave radiation (Garrett et al. 2010; Ravichandran, Meiburg \& Govindarajan 2020). The coupling of the cloud bottom to the cloud-top instability is also an important topic.

Supplementary material. The supplemental material contains the eigenvalue solver, nonlinear spectral model, all the plotting codes and the detailed math derivation; it is available at https://doi.org/10.1017/jfm. 2021.784. 


\section{Saturation interface instability}

Acknowledgements. The author is grateful for Professor M. O'Neill at Stanford University for fruitful discussion and proofreading. The early idea of using the delta function to represent radiative cooling at the saturation interface came when the author was a master's student at IAP/CAS. The establishment of the theoretical framework started during an individual class project at Stanford University ME 451B class, instructed by Professor S. Lele. Professor L. Thomas at Stanford University, Professor J. Mellado at the Max-Planck Institute for Meteorology and Professor Y. Lin at the Institute of Atmospheric Physics, Chinese Academy of Sciences, provided valuable guidance. Three anonymous reviewers provided valuable comments that significantly improved the manuscript.

Declaration of interests. The author reports no conflict of interest.

Author ORCIDs.

(i) Hao Fu https://orcid.org/0000-0002-9524-0001.

\section{Appendix A. The representation of radiative cooling}

In this appendix a physical interpretation of the constant radiative cooling rate formulation used in (2.6) is presented. The non-uniform radiative cooling at the interface due to temperature difference is shown to be a very weak stabilizing factor.

Only longwave radiation is explicitly considered. The solar radiation causes heating deep inside the saturated layer (Oliver, Lewellen \& Williamson 1978). It is qualitatively considered as a part of the fixed temperature boundary condition at $z^{*}=-H$ which is a boundary heat source. The sharp longwave absorption coefficient contrast between the cloudy and clear air makes the radiative flux diverge near the cloud top, causing radiative cooling that is concentrated near the saturation interface $z_{s}^{*}\left(x^{*}, y^{*}, t^{*}\right)$ (Larson, Kotenberg \& Wood 2007; de Lozar \& Mellado 2013). The typical longwave radiation penetration depth at the stratocumulus top is around $15 \mathrm{~m}$ (de Lozar \& Mellado 2013). If the longwave radiation penetration depth in the saturated layer is much shorter than its depth, the saturation interface can be regarded as a blackbody. If we further assume the unsaturated layer is transparent, the radiative cooling can be represented as a Dirac-delta function cooling concentrated at the saturation interface that obeys the Stefan-Boltzmann law,

$$
\left(\frac{\partial T^{*}}{\partial t^{*}}\right)_{\text {rad }} \approx Q_{\text {rad }}^{*} \delta\left(z^{*}-z_{s}^{*}\right) \quad \text { with } Q_{r a d}^{*} \approx-\frac{\sigma_{S} T^{* 4}}{\rho_{0} c_{p}} .
$$

Here $Q_{\text {rad }}^{*}$ is a constant radiative flux density expressed in temperature (negative, unit: $\left.\mathrm{K} \mathrm{s}^{-1} \mathrm{~m}^{-2}\right), \sigma_{S} \approx 5.67 \times 10^{-8} \mathrm{~W} \mathrm{~m}^{-2} \mathrm{~K}^{-4}$ is the Stefan-Boltzmann constant. The motivation of using the Dirac-delta function is to make the problem more analytically tractable. Equation (A1) is straightforward for radiative cooling in the unsaturated layer. The radiative cooling in the saturated layer is slower due to the compensation by condensation heating (de Lozar \& Mellado 2015). However, the extra liquid water caused by radiative cooling, which is close to the interface, is swiftly evaporated by diffusion in this laminar problem. Thus, the net cooling is the same.

In the turbulent regime, de Lozar \& Mellado (2013) set a horizontally uniform radiative cooling, which neglects both the undulation of the radiative cooling source and the inhomogeneity of the radiative flux density at the interface. This can be regarded as the fixed TRBC test in the turbulent regime. They invoked the Stefan-Boltzmann law to explain that the characteristic horizontal temperature difference at the interface of a real cloud is too small to produce any difference in emission, and the eddy mixing is fast enough to eliminate the tiny cooling difference. This physical judgment is verified by de Lozar \& Mellado (2015) who included both effects in their simulation and found no significant difference. Though the scaling argument by de Lozar \& Mellado (2013) is 


\section{H. Fu}

reasonable for the turbulent regime, it does not tell whether they should be neglected for the small-amplitude instability problem where eddy mixing is absent and any perturbation is small compared with the basic state.

In the main body text, the undulation of radiative source is shown to be important at least within this model. The validity of using a constant $Q_{\mathrm{rad}}$ (uniform radiative cooling at the interface) is discussed here. For small temperature perturbation at the interface, the non-dimensional perturbation radiative cooling $Q_{\text {rad }}^{\prime}$ is proportional to the non-dimensional temperature anomaly $T^{\prime}$. The ratio of $Q_{\text {rad }}^{\prime}$ to the basic state constant radiative cooling rate $Q_{\text {rad }}$ is

$$
\frac{Q_{\text {rad }}^{\prime}}{Q_{\text {rad }}} \approx \frac{4 \Delta T}{T_{0}}\left(\left.T\right|_{z=z_{s}}-\left.\bar{T}\right|_{z=0}\right) \approx \frac{4 \Delta T}{T_{0}}\left(\left.T^{\prime}\right|_{z=0^{ \pm}}+\left.z_{s} \frac{\mathrm{d} \bar{T}}{\mathrm{~d} z}\right|_{z=0^{ \pm}}\right)
$$

where we have used the linearized form of the Stefan-Boltzmann law: $\sigma_{S} T^{* 4} \approx \sigma_{S} T_{0}^{4}[1+$ $\left.4\left(T^{*}-T_{0}\right) / T_{0}\right]$, and the ' \pm ' denote $z=0^{-}$and $z=0^{+}$quantities. The effect of non-uniform radiative cooling appears in the heat flux matching condition. Equation (3.11) is replaced with

$$
\left.D \hat{T}\right|_{z=0^{+}}-\left.D \hat{T}\right|_{z=0^{-}}=-M\left(\left.D \hat{q}_{t}\right|_{z=0}-\left.\lambda D \hat{T}\right|_{z=0^{-}}\right)-\operatorname{Pr} \hat{Q}_{r a d}
$$

where $\hat{Q}_{\text {rad }}$ is defined as the normal mode amplitude of $Q_{\text {rad }}^{\prime}=\hat{Q}_{\text {rad }} \exp \left[\mathrm{i}\left(k_{x} x+\right.\right.$ $\left.\left.k_{y} y\right)\right] \exp (\sigma t)$. After using the normal mode form of the Clausius-Clapeyron equation (2.20) to express the interfacial temperature with water content in (A2), we get

$$
\hat{Q}_{\text {rad }}=Q_{\text {rad }} \frac{4 \Delta T}{T_{0}} \frac{\left.\hat{q}_{t}\right|_{z=0}-\hat{z}_{s}}{\lambda} .
$$

In $\S 4.3,\left.T\right|_{z=z_{s}}$ has been shown to be larger than $\left.\bar{T}\right|_{z=0}$ at the updraft $\left(\left.\hat{q}_{t}\right|_{z=0} \geq \hat{z}_{s}\right.$ using the Clausius-Clapeyron equation), so the temperature is anomalously high and the radiative cooling is anomalously strong. This is a stabilizing factor, in contrary to the radiative effect on the undulating interface which is a destabilizing effect.

The relative contribution of the non-uniform radiative cooling and the undulating radiative cooling source is estimated here. The correlation between the forcing term and $w$ is used to quantify the contribution of these two effects to buoyancy flux tendency (opposite in sign), a method that has been introduced in $\$ 4.5$. The buoyancy flux tendency contributed by non-uniform radiative cooling (NR) is denoted as $\overline{w(\partial T / \partial t)_{N R}}$, and that by undulating radiative source (UR) is $\overline{w(\partial T / \partial t)_{U R}}$. The magnitude of their ratio, with an imposed minus sign, is

$$
\begin{aligned}
-\frac{\int_{-1}^{\infty} \overline{w\left(\frac{\partial T}{\partial t}\right)_{N R} \mathrm{~d} z}}{\int_{-1}^{\infty} \overline{w\left(\frac{\partial T}{\partial t}\right)_{U R} \mathrm{~d} z}} & =-\frac{\int_{-1}^{\infty} \hat{w} \hat{Q}_{\text {rad }} \delta(z) \mathrm{d} z}{\int_{-1}^{\infty} \hat{w}\left(-Q_{\text {rad }}\right) \hat{z}_{s} \frac{\mathrm{d} \delta(z)}{\mathrm{d} z} \mathrm{~d} z} \\
& =-\frac{\left.\hat{Q}_{\text {rad }} \hat{w}\right|_{z=0}}{\left.Q_{\text {rad }} \hat{z}_{s} D \hat{w}\right|_{z=0}}
\end{aligned}
$$




\section{Saturation interface instability}

$$
\begin{aligned}
& \lesssim \frac{4 \Delta T / T_{0}}{\hat{z}_{s}} \frac{1}{\lambda}\left(\left.\hat{q}_{t}\right|_{z=0}-\hat{z}_{s}\right) \\
& \lesssim \frac{4 \Delta T}{T_{0}}\left(-\gamma_{T}\right) .
\end{aligned}
$$

Here we have used (A4) to express $\hat{Q}_{\text {rad }}$, used (4.1) to constrain $\hat{z}_{s}$, and used $\left.\hat{w}\right|_{z=0} \lesssim$ $-\left.D \hat{w}\right|_{z=0}$ because $-\left.D \hat{w}\right|_{z=0}$ scales as the maximum $\hat{w}$ in the saturated layer. Thus, a larger temperature drop $\Delta T$ across the unstable layer and a larger stratification ratio magnitude $-\gamma_{T}$ make the non-uniform radiative cooling at the interface more significant.

The parameter $\Delta T=1.5 \mathrm{~K}, T_{0}=285 \mathrm{~K}$ and $\gamma_{T}=-2.5$ lead to $-4 \gamma_{T} \Delta T / T_{0} \approx 0.05$, which means the non-uniform radiative cooling is negligible. This is verified in figure 3( $a)$ where the solid black line (this effect omitted) and the dashed green line (this effect retained) almost coincide.

\section{Appendix B. The solution procedure of the eigenvalue problem}

The interface undulation, non-uniform evaporation and non-uniform radiation (introduced in Appendix A) are considered. Substituting the eigenfunctions in (3.4) into the matching condition in (3.5), (3.9) and (A3), we obtain six equations which render a homogeneous linear system with $\boldsymbol{A}$ as the coefficient matrix and the amplitude coefficients $f$ as the solution vector,

$$
\boldsymbol{A}=\left[\begin{array}{cccccc}
\sinh \left(p_{1}\right) & \sinh \left(p_{2}\right) & \sinh \left(p_{3}\right) & 1 & 1 & 1 \\
p_{1} \cosh \left(p_{1}\right) & p_{2} \cosh \left(p_{2}\right) & p_{3} \cosh \left(p_{3}\right) & -p_{4} & -p_{5} & -p_{6} \\
p_{1}^{2} \sinh \left(p_{1}\right) & p_{2}^{2} \sinh \left(p_{2}\right) & p_{3}^{2} \sinh \left(p_{3}\right) & p_{4}^{2} & p_{5}^{2} & p_{6}^{2} \\
p_{1}^{3} \cosh \left(p_{1}\right) & p_{2}^{3} \cosh \left(p_{2}\right) & p_{3}^{3} \cosh \left(p_{3}\right) & -p_{4}^{3} & -p_{5}^{3} & -p_{6}^{3} \\
E_{1} & E_{2} & E_{3} & E_{4} & E_{5} & E_{6} \\
F_{1} & F_{2} & F_{3} & F_{4} & F_{5} & F_{6}
\end{array}\right],
$$

where ' []$^{\mathrm{T}}$ ' means transpose. The $\hat{T}$ matching condition is

$$
E_{m}= \begin{cases}\left(p_{m}^{2}-K^{2}-\sigma\right)\left(p_{m}^{2}-K^{2}\right) \sinh \left(p_{m}\right) \frac{P r}{R a K^{2}}+\eta_{T}\left(\left.\hat{q}_{t}\right|_{z=0}\right)_{m}, & m=1,2,3, \\ \left(p_{m}^{2}-K^{2}-\sigma\right)\left(p_{m}^{2}-K^{2}\right) \frac{P r}{R a K^{2}} \frac{1-\lambda}{1-\lambda \gamma_{T}}-\eta_{T}\left(\left.\hat{q}_{t}\right|_{z=0}\right)_{m}, & m=4,5,6 .\end{cases}
$$

The $D \hat{T}$ matching condition is

$$
F_{m}= \begin{cases}\left(p_{m}^{2}-K^{2}-\sigma\right)\left(p_{m}^{2}-K^{2}\right) p_{m} \cosh \left(p_{m}\right) \frac{\operatorname{Pr}}{\operatorname{RaK^{2}}(1+\lambda M)} & \\ -M\left(\left.D \hat{q}_{t}\right|_{z=0}\right)_{m}-4 \operatorname{Pr} \frac{\Delta T}{T_{0}} Q_{r a d} \frac{\left(\left.\hat{q}_{t}\right|_{z=0}\right)_{m}-\hat{z}_{s}}{\lambda}, & m=1,2,3, \\ -\left(p_{m}^{2}-K^{2}-\sigma\right)\left(p_{m}^{2}-K^{2}\right) p_{m} \frac{\operatorname{Pr}}{\operatorname{Ra} K^{2}} & \\ \quad+M\left(\left.D \hat{q}_{t}\right|_{z=0}\right)_{m}+4 \operatorname{Pr} \frac{\Delta T}{T_{0}} Q_{r a d} \frac{\left(\left.\hat{q}_{t}\right|_{z=0}\right)_{m}-\hat{z}_{s}}{\lambda}, & m=4,5,6 .\end{cases}
$$




\section{H. Fu}

Here $\left(\left.\hat{q}_{t}\right|_{z=0}\right)_{m}$ and $\left(\left.D \hat{q}_{t}\right|_{z=0}\right)_{m}$ denote the projection of $\left.\hat{q}_{t}\right|_{z=0}$ and $\left.D \hat{q}_{t}\right|_{z=0}$ on the $m$ th eigenfunction, and their expressions are shown in Appendix C. For the fixed TRBC, the matching conditions are $\left.\hat{T}\right|_{z=0^{-}}=\left.\hat{T}\right|_{z=0^{+}}$and $\left.D \hat{T}\right|_{z=0^{-}}=\left.D \hat{T}\right|_{z=0^{+}}$, and the $E_{m}$ and $F_{m}$ (denoted as $\left(E_{m}\right)_{\text {fixed }}$ and $\left.\left(F_{m}\right)_{\text {fixed }}\right)$ are

$$
\begin{gathered}
\left(E_{m}\right)_{\text {fixed }}= \begin{cases}\left(p_{m}^{2}-K^{2}-\sigma\right)\left(p_{m}^{2}-K^{2}\right) \sinh \left(p_{m}\right) \frac{\operatorname{Pr}}{\operatorname{Ra} K^{2}}, & m=1,2,3, \\
\left(p_{m}^{2}-K^{2}-\sigma\right)\left(p_{m}^{2}-K^{2}\right) \frac{\operatorname{Pr}}{\operatorname{Ra} K^{2}}, & m=4,5,6,\end{cases} \\
\left(F_{m}\right)_{\text {fixed }}= \begin{cases}\left(p_{m}^{2}-K^{2}-\sigma\right)\left(p_{m}^{2}-K^{2}\right) p_{m} \cosh \left(p_{m}\right) \frac{\operatorname{Pr}}{\operatorname{Ra} K^{2}}, & m=1,2,3, \\
-\left(p_{m}^{2}-K^{2}-\sigma\right)\left(p_{m}^{2}-K^{2}\right) p_{m} \frac{\operatorname{Pr}}{R a K^{2}}, & m=4,5,6 .\end{cases}
\end{gathered}
$$

The solution of the eigenvalue problem corresponds to $\operatorname{det}(\boldsymbol{A})=0$ (determinant is zero). It is numerically solved by minimizing $|\operatorname{det}(\boldsymbol{A})|$ on $\sigma_{r}$ axis $\left(\sigma_{i}=0\right)$. The solver has been benchmarked with the fixed TRBC marginal curves shown in figure 3 of the paper of Ogura \& Kondo (1970).

\section{Appendix C. The solution of the advection-diffusion equation of $\hat{q}_{t}$}

The general solution of $\hat{q}_{t}$ can be written as

$$
\hat{q}_{t}(z)=C_{1}(z) \exp (\mu z)+C_{2}(z) \exp (-\mu z), \quad \text { with } \mu=\left(K^{2}+\sigma P r\right)^{1 / 2} .
$$

As $\hat{w}$ is piecewise at the lower and upper layers, $C_{1}(z)$ and $C_{2}(z)$ are also piecewise:

$$
\begin{aligned}
C_{1}(z)= \begin{cases}\sum_{m=1}^{6} a_{m}\left[M_{1 m}(z)+K_{1 m}\right], & -1<z \leq 0, \\
\sum_{m=1}^{6} a_{m}\left[N_{1 m}(z)+M_{1 m}(0)+K_{1 m}\right], & 0 \leq z<\infty,\end{cases} \\
C_{2}(z)= \begin{cases}\sum_{m=1}^{6} a_{m}\left[M_{2 m}(z)+K_{2 m}\right], & -1<z \leq 0, \\
\sum_{m=1}^{6} a_{m}\left[N_{2 m}(z)+M_{2 m}(0)+K_{2 m}\right], & 0 \leq z<\infty,\end{cases}
\end{aligned}
$$

where the functions $M_{1 m}(z), M_{2 m}(z), N_{1 m}(z)$ and $N_{2 m}(z)$ are

$$
M_{1 m}(z)= \begin{cases}-\frac{P r}{4 \mu} \exp \left(p_{m}\right) \frac{\exp \left[\left(p_{m}-\mu\right) z\right]-\exp \left[-\left(p_{m}-\mu\right)\right]}{p_{m}-\mu} & \\ -\frac{P r}{4 \mu} \exp \left(-p_{m}\right) \frac{\exp \left[-\left(p_{m}+\mu\right) z\right]-\exp \left(p_{m}+\mu\right)}{p_{m}+\mu}, & m=1,2,3, \\ 0, & m=4,5,6,\end{cases}
$$




$$
M_{2 m}(z)= \begin{cases}\frac{P r}{4 \mu} \exp \left(p_{m}\right) \frac{\exp \left[\left(p_{m}+\mu\right) z\right]-\exp \left[-\left(p_{m}+\mu\right)\right]}{p_{m}+\mu} & \\ \quad+\frac{P r}{4 \mu} \exp \left(-p_{m}\right) \frac{\exp \left[-\left(p_{m}-\mu\right) z\right]-\exp \left(p_{m}-\mu\right)}{p_{m}-\mu}, & m=1,2,3, \\ 0, & m=4,5,6,\end{cases}
$$

$$
\begin{gathered}
N_{1 m}(z)= \begin{cases}0, & m=1,2,3, \\
\frac{\operatorname{Pr}}{2 \mu} \frac{\exp \left[-\left(p_{m}+\mu\right) z\right]-1}{p_{m}+\mu}, & m=4,5,6,\end{cases} \\
N_{2 m}(z)= \begin{cases}0, & m=1,2,3, \\
-\frac{\operatorname{Pr}}{2 \mu} \frac{\exp \left[-\left(p_{m}-\mu\right) z\right]-1}{p_{m}-\mu}, & m=4,5,6 .\end{cases}
\end{gathered}
$$

The constants $K_{1 m}$ and $K_{2 m}$ are determined by imposing the lower and upper boundary condition $\left.\hat{q}_{t}\right|_{z=-1}=0$ and $\left.\hat{q}_{t}\right|_{z \rightarrow \infty}=0$ on each of the $m=1,2,3,4,5,6$ mode independently, and their expressions are

$$
\begin{gathered}
K_{1 m}= \begin{cases}-M_{1 m}(0), & m=1,2,3, \\
\frac{\operatorname{Pr}}{2 \mu} \frac{1}{p_{m}+\mu}, & m=4,5,6,\end{cases} \\
K_{2 m}=-K_{1 m} \exp (-2 \mu), \quad m=1,2,3,4,5,6 .
\end{gathered}
$$

The $\hat{q}_{t}$ and $D \hat{q}_{t}$ at $z=0$ will be used to calculate the matching condition in (B3) and (B4). Their projections on the $m$ th mode are denoted as $\left(\left.\hat{q}_{t}\right|_{z=0}\right)_{m}$ and $\left(\left.D \hat{q}_{t}\right|_{z=0}\right)_{m}$ :

$$
\begin{gathered}
\left(\left.\hat{q}_{t}\right|_{z=0}\right)_{m}=K_{1 m}+K_{2 m}+M_{1 m}(0)+M_{2 m}(0), \\
\left(\left.D \hat{q}_{t}\right|_{z=0}\right)_{m}=\mu\left[K_{1 m}-K_{2 m}+M_{1 m}(0)-M_{2 m}(0)\right] .
\end{gathered}
$$

\section{Appendix D. The purely evaporative cooling case}

This appendix shows that without radiative cooling, the marginal stability curve fully overlaps with the corresponding fixed TRBC problem.

The source-free advective-diffusive evolution of enthalpy $h^{*}$ and $q_{t}^{*}$ in non-precipitating moist Rayleigh-Bénard convection with constant diffusivity enables us to express $q_{t}^{*}$ with $T^{*}$ (Bretherton 1987), without invoking the direct solution of (3.10). This is an important relation that can simplify the stability analysis, so we re-derive it below with the thermodynamic framework of this paper. The expression of $h^{*}$ is

$$
h^{*}=c_{p} T^{*}+L_{v} q_{v}^{*}= \begin{cases}c_{p} T^{*}+L_{v} q_{t}^{*}, & z_{s} \leq z<\infty, \\ c_{p} T^{*}+L_{v}\left[q_{v s 0}+\tilde{\lambda}\left(T^{*}-T_{0}\right)\right], & -1<z \leq z_{s} .\end{cases}
$$




\section{H. Fu}

The non-dimensional enthalpy $h$, its basic state profile $\bar{h}$ and the disturbance $h^{\prime}$ are

$$
\begin{gathered}
h=\frac{h^{*}-\left(c_{p} T_{0}+L_{v} q_{v s 0}\right)}{L_{v} \Delta q_{t}}, \\
\bar{h}=\frac{-c_{p} \Delta T \gamma_{T} z-L_{v} \Delta q_{t} z}{L_{v} \Delta q_{t}}=-\left(\frac{\gamma_{T}}{M}+1\right) z, \\
h^{\prime}= \begin{cases}\frac{c_{p} \Delta T T^{\prime}+L_{v} \Delta q_{t} q_{t}^{\prime}}{L_{v} \Delta q_{t}}=\frac{T^{\prime}}{M}+q_{t}^{\prime}, & 0 \leq z<\infty, \\
\frac{c_{p} \Delta T T^{\prime}+L_{v} \tilde{\lambda} \Delta T T^{\prime}}{L_{v} \Delta q_{t}}=T^{\prime}\left(\frac{1}{M}+\lambda\right), & -1<z \leq 0,\end{cases}
\end{gathered}
$$

where $\bar{h}$ is a linear function of $z$ in the whole depth. The linearized governing equation and boundary condition of $h^{\prime}$ and $q_{t}^{\prime}$ are

$$
\left.\begin{array}{lll}
\frac{\partial h^{\prime}}{\partial t}+w \frac{\mathrm{d} \bar{h}}{\mathrm{~d} z}=\frac{1}{P r} \nabla^{2} h^{\prime}, & \text { with }\left.h^{\prime}\right|_{z=-1}=0, & \left.h^{\prime}\right|_{z \rightarrow \infty}=0, \\
\frac{\partial q_{t}^{\prime}}{\partial t}+w \frac{\mathrm{d} \overline{q_{t}}}{\mathrm{~d} z}=\frac{1}{\operatorname{Pr}} \nabla^{2} q_{t}^{\prime}, & \text { with }\left.q_{t}^{\prime}\right|_{z=-1}=0, & \left.q_{t}^{\prime}\right|_{z \rightarrow \infty}=0 .
\end{array}\right\}
$$

Equation (D5) renders the relationship between $h^{\prime}$ and $q_{t}^{\prime}$ as follows:

$$
\frac{h^{\prime}}{\bar{q}_{t}^{\prime}}=\frac{\mathrm{d} \bar{h} / \mathrm{d} z}{\mathrm{~d} \overline{q_{t}} / \mathrm{d} z}=\frac{\gamma_{T}}{M}+1 .
$$

Substituting (D4) into (D6), and using (2.16), the relationship between $T^{\prime}$ and $q_{t}^{\prime}$ is obtained:

$$
T^{\prime}= \begin{cases}\gamma_{T} q_{t}^{\prime}, & 0<z<\infty, \\ q_{t}^{\prime}, & -1<z<0 .\end{cases}
$$

At $z=0^{+}$, (D7) renders $\left.\hat{T}\right|_{z=0^{+}}=\left.\gamma_{T} \hat{q}_{t}\right|_{z=0}$. Substituting it into (3.7), we get

$$
\hat{z}_{s}=\left.\hat{q}_{t}\right|_{z=0}
$$

It indicates that the interface moves with a $q_{t}$ contour surface. Because there is $q_{t}=q_{v s}=$ $\lambda T$ at the saturation interface, the saturation interface is also a temperature contour surface. This property, which is still valid in the nonlinear regime, has been pointed out by Mellado et al. (2009) who used an equivalent governing equation expressed with mixing fraction rather than $T$ and $q_{t}$.

Equation (D7) and the continuity of $q_{t}^{\prime}$ at $z=0$ are powerful constraints, which render the relationship between $\left.\hat{T}\right|_{z=0^{-}}$and $\left.\hat{T}\right|_{z=0^{+}}$, as well as $\left.D \hat{T}\right|_{z=0^{-}}$and $\left.D \hat{T}\right|_{z=0^{+}}$as follows:

$$
\left.\hat{T}\right|_{z=0^{+}}=\left.\gamma_{T} \hat{T}\right|_{z=0^{-}},\left.\quad D \hat{T}\right|_{z=0^{+}}=\left.\gamma_{T} D \hat{T}\right|_{z=0^{-}} .
$$

With the help of (3.4) and (3.8), (D9) can replace the lower two rows of the matrix $\boldsymbol{A}$ in (B1) with

$$
E_{m}= \begin{cases}\gamma_{T} \frac{\operatorname{Pr}}{\operatorname{RaK} K^{2}}\left(p_{m}^{2}-K^{2}\right)\left(p_{m}^{2}-K^{2}-\sigma\right) \sinh \left(p_{m}\right)=\frac{-\operatorname{Pr} \sinh \left(p_{m}\right)}{p_{m}^{2}-K^{2}-\operatorname{Pr} \sigma}, & m=1,2,3, \\ \frac{\operatorname{Pr}}{R a K^{2}}\left(p_{m}^{2}-K^{2}\right)\left(p_{m}^{2}-K^{2}-\sigma\right)=\frac{-\operatorname{Pr}}{p_{m}^{2}-K^{2}-\operatorname{Pr} \sigma}, & m=4,5,6,\end{cases}
$$




\section{Saturation interface instability}

$$
F_{m}= \begin{cases}\gamma_{T} \frac{\operatorname{Pr}}{\operatorname{Ra} K^{2}}\left(p_{m}^{2}-K^{2}\right)\left(p_{m}^{2}-K^{2}-\sigma\right) p_{m} \cosh \left(p_{m}\right)=\frac{-\operatorname{Pr} p_{m} \cosh \left(p_{m}\right)}{p_{m}^{2}-K^{2}-\operatorname{Pr} \sigma}, & m=1,2,3, \\ \frac{\operatorname{Pr}}{\operatorname{Ra} K^{2}}\left(p_{m}^{2}-K^{2}\right)\left(p_{m}^{2}-K^{2}-\sigma\right) p_{m}=\frac{\operatorname{Pr} p_{m}}{p_{m}^{2}-K^{2}-\operatorname{Pr} \sigma}, & m=4,5,6 .\end{cases}
$$

The last equality of each line of the equations has used the sixth-order ODE (3.2). The modified matrix $\boldsymbol{A}$ with the new $E_{m}$ and $F_{m}$ is defined as $\tilde{\boldsymbol{A}}$, and the new amplitude vector is $\tilde{f}$. Here we show that the free TRBC in this case has the identical marginal stability curve with the fixed TRBC. The idea is to manipulate $\tilde{\boldsymbol{A}}$ to show that for given $R a$ and $K$ (and therefore $\left.p_{m}\right), \operatorname{det}(\boldsymbol{A})=0$ and $\operatorname{det}(\tilde{\boldsymbol{A}})=0$ occur at the same time. The proof procedure is shown below.

(i) First, we introduce a diagonal matrix $\boldsymbol{B}=\operatorname{diag}\left\{\left(p_{m}^{2}-K^{2}-\operatorname{Pr} \sigma\right)\right\}$, and rewrite $\tilde{\boldsymbol{A}} \tilde{\boldsymbol{f}}=0$ as $\tilde{\boldsymbol{A}} \boldsymbol{B} \boldsymbol{B}^{-1} \tilde{\boldsymbol{f}}=0$.

(ii) Second, we note that the fifth and sixth row of $\tilde{\boldsymbol{A}} \boldsymbol{B}$ is identical to the original first and second line of $\tilde{\boldsymbol{A}}$. Some row transformations on $\tilde{\boldsymbol{A}} \boldsymbol{B}$ can transform it to $\boldsymbol{A}$, so $\tilde{\boldsymbol{A}} \boldsymbol{B}$ and $\boldsymbol{A}$ share the row space and have the same rank. As $\boldsymbol{B}$ is non-singular, $\tilde{\boldsymbol{A}}$ and $\boldsymbol{A}$ always have the same $\operatorname{rank}, \operatorname{so} \operatorname{det}(\boldsymbol{A})=0$ and $\operatorname{det}(\tilde{\boldsymbol{A}})=0$ must occur at the same time.

This indicates $\tilde{\boldsymbol{f}}=\boldsymbol{B} \boldsymbol{f}$. It means the only difference between the fixed TRBC and the free TRBC is the amplitude coefficients, which makes the eigenfunctions (e.g. $\hat{w}$ ) different.

Figure 4 shows that, compared with the Ref-F test, the primary lower cell in the Ref-E test is more confined in the lower layer, and the secondary upper cell is stronger. This can be understood with $\tilde{f}=\boldsymbol{B} \boldsymbol{f}$. For the neutral mode where $\sigma=0, \boldsymbol{B}$ can be viewed as a $\nabla^{2}$ operator which acts on the eigenfunction of the fixed TRBC. As $\nabla^{2}$ means coarsening, the eigenfunction of the free TRBC should be curvier.

As the marginal curve of the free and fixed TRBC always collapse, the candidate $\sigma$ for any given $R a$ should be identical. Thus, the principle of exchange of stability which applies for the fixed TRBC (Whitehead 1968) also applies for this purely evaporative case. Note that the relation shown in this appendix also works for a growing mode.

\section{REFERENCES}

Austin, P. 1995 A linear stability analysis of stratocumulus convection driven by radiative cooling. Tellus A 47 (2), 259-274.

Bois, P.-A. \& Kubicki, A. 2003 A theoretical model for double diffusive phenomena in cloudy convection. In Annales Geophysicae (ed. I.P. Duvel), vol. 21, pp. 2201-2201. Copernicus GmbH.

BREIDENTHAL, R.E. \& BAKER, M.B. 1985 Convection and entrainment across stratified interfaces. J. Geophys. Res. Atmos. 90 (D7), 13055-13062.

BRETHERTON, C.S. 1987 A theory for nonprecipitating moist convection between two parallel plates. Part I: thermodynamics and "linear" solutions. J. Atmos. Sci. 44 (14), 1809-1827.

Bretherton, C.S., Blossey, P.N. \& UChida, J. 2007 Cloud droplet sedimentation, entrainment efficiency, and subtropical stratocumulus albedo. Geophys. Res. Lett. 34 (3), L03813.

Busse, F.H. \& Schubert, G. 1971 Convection in a fluid with two phases. J. Fluid Mech. 46 (4), 801-812.

Chandrakar, K.K., Cantrell, W., Krueger, S., Shaw, R.A. \& Wunsch, S. 2020 Supersaturation fluctuations in moist turbulent Rayleigh-Bénard convection: a two-scalar transport problem. J. Fluid Mech. 884, A19.

Cotton, W.R., Bryan, G. \& VAn Den Heever, S.C. 2011 Fogs and stratocumulus clouds. In International Geophysics (ed. W. Cotton, G. Bryan \& S. van den Heever), vol. 99, pp. 179-242. Elsevier.

DE LozAR, A. \& Mellado, J.P. 2013 Direct numerical simulations of a smoke cloud-top mixing layer as a model for stratocumuli. J. Atmos. Sci. 70 (8), 2356-2375. 


\section{H. Fu}

DE Lozar, A. \& Mellado, J.P. 2015 Mixing driven by radiative and evaporative cooling at the stratocumulus top. J. Atmos. Sci. 72 (12), 4681-4700.

DurRan, D.R. 2010 Numerical Methods for Fluid Dynamics: With Applications to Geophysics, vol. 32. Springer Science \& Business Media.

Emanuel, K.A. 1994 Atmospheric Convection. Oxford University Press.

FIELDER, B.H. 1984 The mesoscale stability of entrainment into cloud-topped mixed layers. J. Atmos. Sci. 41 (1), 92-101.

Garrett, T.J., Schmidt, C.T., Kihlgren, S. \& Cornet, C. 2010 Mammatus clouds as a response to cloud-base radiative heating. J. Atmos. Sci. 67 (12), 3891-3903.

Gribov, V.N. \& GUREVICH, L.E. 1957 On the theory of the stability of a layer located at a superadiabatic temperature gradient in a gravitational filed. Sov. Phys. JETP 4, 720-729.

HSIEH, D.Y. 1972 Effects of heat and mass transfer on Rayleigh-Taylor instability. Trans. ASME J. Basic Engng 94 (1), 156-160.

Konovalov, V.V., Lyubimov, D.V. \& Lyubimova, T.P. 2017 Influence of phase transition on the instability of a liquid-vapor interface in a gravitational field. Phys. Rev. Fluids 2 (6), 063902.

Lange, M., Zühlke, D., Holz, O., Villmann, T. \& Mittweida, S.-G. 2014 Applications of lp-norms and their smooth approximations for gradient based learning vector quantization. In ESANN 2014 proceedings, European Symposium on Artificial Neural Networks, Computational Intelligence and Machine Learning, pp. 271-276.

Larson, V.E., KotenberG, K.E. \& Wood, N.B. 2007 An analytic longwave radiation formula for liquid layer clouds. Mon. Weath. Rev. 135 (2), 689-699.

LebedeV, V.I. 1991 On formulae for roots of cubic equation. Russ. J. Numer. Anal. Math. Model. 6 (4), 315-324.

LILLY, D.K. 1968 Models of cloud-topped mixed layers under a strong inversion. Q. J. R. Meteorol. Soc. 94 (401), 292-309.

McFadden, G.B., Coriell, S.R., Gurski, K.F. \& Cotrell, D.L. 2007 Onset of convection in two liquid layers with phase change. Phys. Fluids 19 (10), 104109.

Mellado, J.P. 2017 Cloud-top entrainment in stratocumulus clouds. Annu. Rev. Fluid Mech. 49, $145-169$.

Mellado GonzÁlez, J.P. 2010 The evaporatively driven cloud-top mixing layer. J. Fluid Mech. 660, 5-36.

Mellado, J.P., Stevens, B., Schmidt, H. \& Peters, N. 2009 Buoyancy reversal in cloud-top mixing layers. Q. J. R. Meteorol. Soc. 135 (641), 963-978.

OGURA, Y. \& Kondo, H. 1970 A linear stability of convective motion in a thermally unstable layer below a stable region. J. Meteorol. Soc. Japan 48 (3), 204-216.

Ogura, Y. \& Yagihashi, A. 1971 Non-stationary finite-amplitude convection in a thin fluid layer bounded by a stably stratified region. J. Atmos. Sci. 28 (8), 1389-1399.

Oliver, D.A., Lewellen, W.S. \& Williamson, G.G. 1978 The interaction between turbulent and radiative transport in the development of fog and low-level stratus. J. Atmos. Sci. 35 (2), 301-316.

Pope, S.B. 2000 Turbulent flows. Cambridge University Press.

Randall, D.A. 1980 Conditional instability of the first kind upside-down. J. Atmos. Sci. 37 (1), $125-130$.

Ravichandran, S., Meiburg, E. \& Govindarajan, R. 2020 Mammatus cloud formation by settling and evaporation. J. Fluid Mech. 899, A27.

SAYLER, B.J. \& BREIDENTHAL, R.E. 1998 Laboratory simulations of radiatively induced entrainment in stratiform clouds. J. Geophys. Res. Atmos. 103 (D8), 8827-8837.

Schneider, T., Kaul, C.M. \& Pressel, K.G. 2019 Possible climate transitions from breakup of stratocumulus decks under greenhouse warming. Nat. Geosci. 12 (3), 163-167.

Schulz, B. \& MEllado, J.P. 2018 Wind shear effects on radiatively and evaporatively driven stratocumulus tops. J. Atmos. Sci. 75 (9), 3245-3263.

Schulz, B. \& Mellado, J.P. 2019 Competing effects of droplet sedimentation and wind shear on entrainment in stratocumulus. J. Adv. Model. Earth Syst. 11 (6), 1830-1846.

Shy, S.S. \& BREIDENTHAL, R.E. 1990 Laboratory experiments on the cloud-top entrainment instability. J. Fluid Mech. 214, 1-15.

Siems, S.T. \& BRETherton, C.S. 1992 A numerical investigation of cloud-top entrainment instability and related experiments. Q. J. R. Meteorol. Soc. 118 (507), 787-818.

Siems, S.T., Bretherton, C.S., BAKer, M.B., Shy, S. \& Breidenthal, R.E. 1990 Buoyancy reversal and cloud-top entrainment instability. Q. J. R. Meteorol. Soc. 116 (493), 705-739.

Spyksma, K., Bartello, P. \& YAU, M.K. 2006 A Boussinesq moist turbulence model. J. Turbul. 7, N32.

Thuburn, J. \& Efstathiou, G.A. 2020 Marginal stability of the convective boundary layer. J. Atmos. Sci. 77 (2), 435-442. 


\section{Saturation interface instability}

TURNeR, J.S. \& YANG, I.K. 1963 Turbulent mixing at the top of stratocumulus clouds. J. Fluid Mech. 17 (2), 212-224.

WhiteHEAD, J.A. 1968 Stability and convection of a thermally destabilized layer of fluid above a stabilized region. $\mathrm{PhD}$ thesis, Yale University.

WhiteheAD, J.A. \& CHEN, M.M. 1970 Thermal instability and convection of a thin fluid layer bounded by a stably stratified region. J. Fluid Mech. 40 (3), 549-576.

YANO, J.-I. 2021 Hydrodynamic stability analysis of CTEI. J. Atmos. Sci. https://hal.archives-ouvertes.fr/hal03215159.

Zhou, B., Simon, J.S. \& ChOw, F.K. 2014 The convective boundary layer in the terra incognita. J. Atmos. Sci. 71 (7), 2545-2563. 